\title{
estudio sobre encofrados de madera modernos
}

Juan M. de la Peña Aznar, Dr. Ingeniero Industrial

$836-15$

\section{sinopsis}

El autor continúa el desarrollo del tema sobre "Encofrados de madera modernos", resumiendo el estudio comparativo - ya hecho en la do el estudio comparativo ya hecho en la Parte III de este Estudio- de las diferentes vigas de madera encolada existentes en e mercado dando, además, las cargas y tensiones admisibles para maderas coniferas y la propuesta de Reglamentación de la Sección de Maderas del Instituto Forestal de Investigaciones y Experiencias de España.

PARTE VII Y ULTIMA

En la parte $\mathbf{V}$ del Estudio del autor, publicada en este artículo, se aborda el importante tema de las colas empleadas para las uniones de madera, algo realmente vital para la obten ción de vigas de madera en celosía simplemente encoladas.

\section{ANALISIS DE LAS TENSIONES DE TRABAJO EN LOS ELEMENTOS Y UNIONES, $Y$ ENSAYOS A ROTURA DE LOS DIVERSOS TIPOS DE VIGAS DE MADERA ENCOLADA PARA ENCOFRADOS MODERNOS}

En esta parte vamos a determinar los esfuerzos o tensiones a que se hallan sometidos los distintos elementos constitutivos de las vigas de madera encolada para encofrados, partiendo de las características de $\mathrm{M}_{a d m}$ y $\mathrm{Q}_{a d m}$ que dan los constructores en sus catálogos, con el fin de comprobar si las tensiones internas están o no dentro de las marcadas en norma.

\section{Vigas de celosía tipo Warren en doble diagonal}

Tomamos como ejemplo la viga STEIDLE-TREGAR de $3,05 \mathrm{~m}$ de longitud, cuyas características (véase Cuadro n. ${ }^{\circ} 3$ de la Parte I publicada en el n. ${ }^{\circ} 318$ de esta Revista) son:

$$
\begin{aligned}
& \mathrm{M}_{a d m}=1.500 \mathrm{~m} \cdot \mathrm{kp} \\
& \mathrm{Q}_{\text {adm }}=1.900 \mathrm{~m} \cdot \mathrm{kp}
\end{aligned}
$$

y en base a las cuales vamos a calcular las tensiones que se originan en sus barras, a efectos de comprobar si cumplen las $\sigma$ y $\tau$ admisibles de la madera empleada, que es una conifera de clase I, según DIN 1052, siendo sus características específicas, respecto a su peso unitario las siguientes:

$$
\begin{gathered}
M_{\text {especifico }}=\frac{1.500}{6,8}=220 \\
\mathrm{Q}_{\text {especifico }}=\frac{1.900}{6,8}=279
\end{gathered}
$$




\subsection{Cordón superior}

Como la viga estudiada tiene una altura a canto total exterior de $360 \mathrm{~mm}$, y sus cordones superior e inferior son iguales y tienen una escuadría aproximada de $60 \times 100 \mathrm{~mm}$, la distancia vertical entre ejes de cordones es de $300 \mathrm{~mm}$, luego el esfuerzo a que está sometido el cordón superior es de:

$$
\frac{1.500}{30} \times 100=5.000 \mathrm{kp}
$$

Como los tableros contrachapados que se apoyan en las vigas se clavan, o mejor, se atornillan a éstas, con objeto de formar paneles de encofrado lo más rígidos posible, podemos considerar como luz de cálculo del cordón superior el que está en contacto con los tableros y sometido a compresión por efecto de la presión enviada por el hormigón fresco, 0,64 m, o sea la distancia entre los ejes geométricos de nudos debidamente corregida para calcular la tensión a que está sometido el cordón superior o comprimido, que trabaja a compresión y flexión simultáneamente.

En el caso del esfuerzo de compresión, dada la continuidad del cordón, adoptaremos la hipótesis de que sus extremos están empotrados - que se acerca bastante a la realidadhabida cuenta de la referida unión de las vigas con los tableros contrachapados del forro del encufrado. Pero además hemos de considerar que los nudos tienen $17 \mathrm{~cm}$ de longitud, de manera que el momento a $8,5 \mathrm{~cm}$ del eje del nudo en una viga empotrada vale:

$$
\begin{aligned}
M_{8,5}= & -\frac{P \cdot l^{2}}{12}+\frac{P \cdot l}{2} \cdot 8,5-\frac{P \cdot 8,5^{2}}{2}= \\
& -\frac{P \cdot 64^{2}}{12}+\frac{P \cdot 64}{2} \cdot 8,5-\frac{P \cdot 8,5^{2}}{2}=105 \cdot \mathrm{cm} \cdot \mathrm{kp}
\end{aligned}
$$

Recordemos por otro lado que la luz límite media de las vigas de madera encolada se calculó en el epígrafe 5 de la Parte I y vale 3,6 m, de donde la carga uniforme que soporta la viga, y en definitiva el cordón superior comprimido es:

$$
\mathrm{q}_{a d m}=\frac{2 \cdot \mathrm{Q}_{a d m}}{3,6}=\frac{2 \times 1.900}{3,6}=1.055 \mathrm{kp} / \mathrm{m}
$$

luego el momento de empotramiento que sufre dicho cordón, en el punto de comienzo real del nudo es:

$$
M_{8,5}=105 \times 10,55=1.108 \mathrm{~cm} \cdot \mathrm{kp}
$$

El área de los cordones, de su sección se entiende, es:

$$
A=10 \times 6=60 \mathrm{~cm}^{2}
$$

y su módulo mínimo resistente:

$$
\mathrm{W}_{\min }=1 / 6 \times 10 \times 6^{2}=60 \mathrm{~cm}^{3}
$$

siendo el momento de inercia mínimo de la sección transversal:

$$
I_{\text {min }}=1 / 12 \times 10 \times 6^{3}=180 \mathrm{~cm}^{4}
$$


luego el radio de giro mínimo valdrá:

$$
\mathrm{i}_{\min }=\frac{180}{60}=1,73 \mathrm{~cm}
$$

y conservando la hipótesis anterior de extremos empotrados, dada la continuidad del cordón y su sujeción al tablero, la luz de cálculo será de $64: 2=32 \mathrm{~cm}$, de modo que la esbelted será:

$$
\lambda \doteq \frac{32}{1,73}=18
$$

para la cual el coeficiente de pandeo $\omega=1,07$, según DIN 1052.

Con todos estos datos ya podemos comprobar la tensión a que está sometido el cordón superior comprimido y flexado, mediante la conocida fórmula:

$$
\sigma=\frac{\mathrm{N} \cdot \omega}{\mathrm{A}}+\frac{\sigma_{c_{u l ! m}}}{\sigma_{t_{u l l m}}} \cdot \frac{\mathrm{M}}{\mathrm{W}_{\min }} \leqslant \sigma_{c_{u d l m}}
$$

y sustituyendo:

$$
\sigma=\frac{5.000 \times 1,07}{60}+0,85 \times \frac{1.108}{60}=105 \mathrm{kp} / \mathrm{cm}^{2} \leqslant \sigma_{c_{a d m}}
$$

redondeando los decimales.

Como la madera empleada para la construcción de estas vigas es pino Norte, de madera blanca exenta de fendas y acebolladuras, casi exenta de nudos y los existentes son pequeños y no saltadizos, se pueden admitir como admisibles para todos los cálculos estáticos de tal madera, los especificados en la Tabla 6 de la Norma DIN 1052 para especies coníferas de clase I, pero disminuidas en 1/6, según apartado 9.4. de dicha Norma, porque se trata de construcciones en madera sometidas a humedad, y aumentadas en un $15 \%$ por tomar cargas principales y suplementarias (apartados 4.1.2. y 9.1.12 de dicha norma). Luego las tensiones admisibles serán:

- Flexión

$$
\begin{aligned}
& \sigma_{u d m}=124 \mathrm{kp} / \mathrm{cm}^{2} \\
& \sigma_{t^{\prime}}=100 \mathrm{~N} \\
& \text { - Compresión paralela a fibras ................................... } \sigma_{c_{c u l m}}=106 \quad "
\end{aligned}
$$

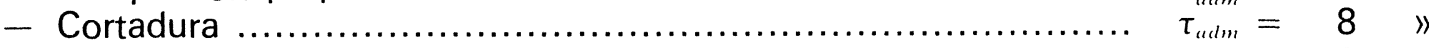$$
\text { - Tracción }
$$$$
\text { - Compresión perpendicular a fibras ............................. } \sigma_{c_{c u l m}}=\begin{array}{r}
19 \\
\tau_{s}
\end{array}
$$

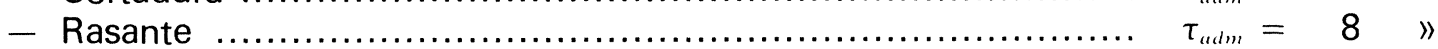

Como se ve la tensión de trabajo del cordón superior, el más cargado por estar sometido a compresión compuesta, es inferior a la máxima admisible minorada por la presencia de la humedad.

\subsection{Cordón inferior}

La carga es de $5.000 \mathrm{kp}$ a tracción en los tramos más cargados, luego su tensión de trabajo bajo el efecto del momento admitido como admisible $(M=1.500 \mathrm{~m} . \mathrm{kp})$ es:

$$
\sigma=\frac{5.000}{60}=83 \mathrm{kp} / \mathrm{cm}^{2}<\sigma_{t_{u d l m}}
$$




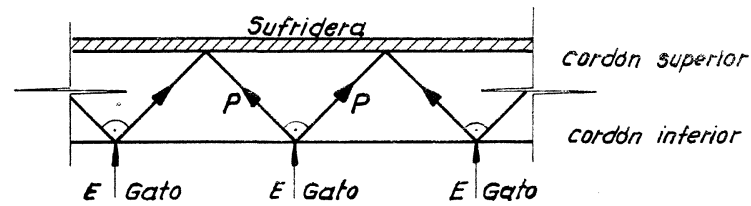

Fig. 1

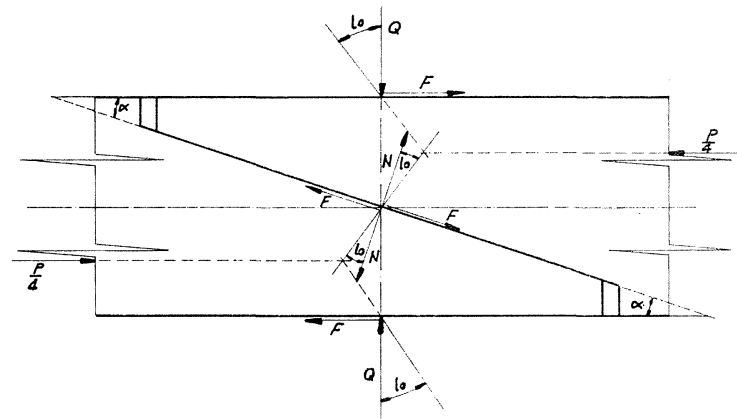

Fig. 2

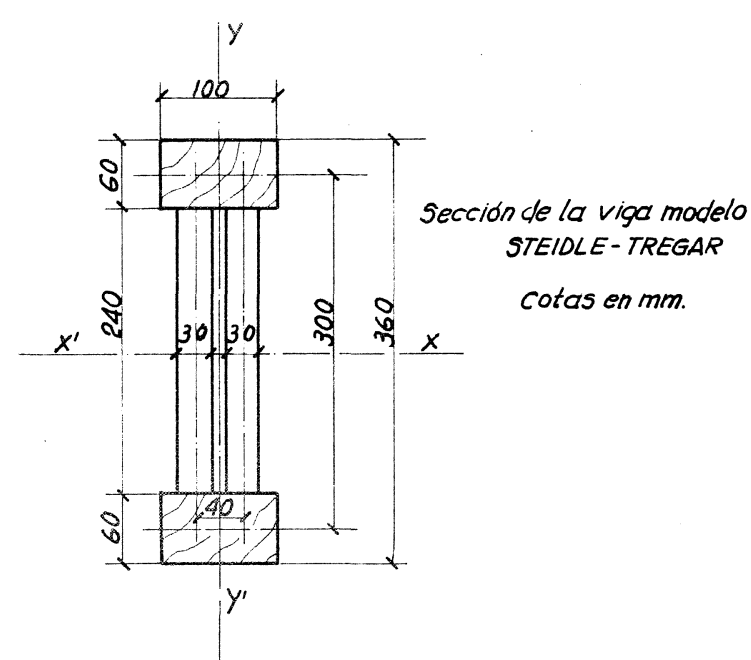

Fig. 3

\subsection{Diagonal comprimida}

La longitud de las diagonales entre ejes de cordones, siendo $300 \mathrm{~mm}$ el canto entre ejes de estos últimos, vale:

$$
300 \times \sqrt{2}=424 \mathrm{~mm}
$$

Por la Fig. 3 se sabe que las diagonales de este tipo de viga que estamos estudiando están formadas por dos escuadrías de $30 \times 60 \mathrm{~mm}$, separados los lados mayores $10 \mathrm{~mm}$, de manera que entre ejes de cada elemento hay una distancia de $40 \mathrm{~mm}$.

Con estos datos podemos ya pasar a calcular las características mecánicas de cada uno de los dos elementos que componen una diagonal, que son:

$$
\begin{aligned}
& A=6 \times 3=18 \mathrm{~cm}^{2} \\
& I_{\text {min }}=1 / 12 \times 6 \times 3^{3}=13,5 \mathrm{~cm}^{4} \\
& W_{\text {min }}=1 / 6 \times 6 \times 3^{2}=9 \mathrm{~cm}^{3} \\
& i=\sqrt{\frac{13,5}{18}}=0,87 \mathrm{~cm}
\end{aligned}
$$

En cuanto a la luz de cálculo a pandeo, tomamos la libre entre caras interiores de cordones de viga, que vale:

$$
l=\frac{360-2 \times 60}{\cos 45^{\circ}}=340 \mathrm{~mm}
$$

luego la esbeltez valdrá:

$$
\lambda=\frac{34}{0,87}=39
$$

y el coeficiente de pandeo, según DIN 1052 será $\omega=1,25$. 


\subsection{Cálculo de los nudos}

Los nudos están formados por espigas en forma de cuña, o entalladuras múltiples normales (ver Fig. 1, Parte 1) con un ángulo de $\alpha=6^{\circ} 30^{\prime}$, y una superficie de contacto entre caras de cuñas o entalladuras de $23,5 \mathrm{~cm}^{2}$. Cada diagonal de la celosía tiene, como ya se ha dicho en este tipo de vigas, dos elementos y cada uno de ellos tiene dos espigas, cuñas o entalladuras que se aparean dos a dos, o sea las dos de un elemento con las otras dos que con aquél forma un ángulo de $90^{\circ}$ en el plano de la viga, y están además en el mismo plano en el espacio, de forma que cada espiga de un elemento conecte con la del otro a través de esos $23,5 \mathrm{~cm}^{2}$ indicados, y ambas penetran en una caja de $10 \mathrm{~mm}$ de ancho labrada en los cordones por sus caras internas.

\subsubsection{Coeficiente de rozamiento}

El coeficiente de rozamiento en reposo de madera de través sobre madera al hilo (las diagonales son perpendiculares entre sí) vale, según Morin, para presión específica reducida del orden de $1 \mathrm{kp} / \mathrm{cm}^{2}, \varphi_{0}=0,34$ (véase Hütte, tomo I, pág. 341 ), luego el ángulo de rozamiento en reposo es:

$$
\varphi_{o}=\operatorname{tg} \varrho_{0}=0,34 \varrho_{0}=18^{\circ} 46^{\prime}
$$

valor muy superior al del ángulo de las espigas o entalladuras de las cuñas que penetran dos a dos en las cajas de los cordones, luego está totalmente asegurada la irreversibilidad del sistema una vez armada la viga incluso sin cola, pues recordemos el principio de la cuña que está en equilibrio dinámico, cuando el ángulo de ella es inferior a dos veces el ángulo de rozamiento, cosa que en este caso se cumple ampliamente pues el ángulo de la cuña ya dijimos que vale $6^{\circ} 30^{\prime}$, y el doble del ángulo de rozamiento en reposo asciende nada menos que a $37^{\circ} 32^{\prime}$.

Por otro lado, según M. P. Parisot del C.T.B. (véase publicación A.I.T.I.M. "Unión de piezas por entalladuras múltiples") el límite máximo de $\varrho_{o}$ es $18^{\circ}$, que coincide muy bien con el valor hallado antes, por lo que adoptaremos como coeficiente de rozamiento de madera sobre madera, seca, con fibras perpendiculares y poca presión de aprieto.

$$
\varrho_{o}=18^{\circ} \text { o sea, } \operatorname{tg} \varrho_{o}=0,32491
$$

\subsubsection{Fuerza de armado}

El armado de las vigas de madera encolada se realiza en una mesa, uno de cuyos lados, correspondiente al cordón superior, está formado por una sufridera con una flecha de 1,7 $\mathrm{mm} / \mathrm{m}$ de longitud, en donde se apoya tal cordón, y en el lado opuesto tantos gatos accionados por aire comprimido como nudos tenga la viga, los cuales aprietan a ella en su plano contra la sufridera, armando asi la viga y comunicando la contraflecha de fabricación que necesite para su trabajo posterior en el campo de las tensiones elásticas.

Tales gatos se componen de cilindros accionados por aire comprimido que, de acuerdo con su diámetro y la presión de aquél, comunican al nudo un empuje de armado de $E=2.000$ $\mathrm{kp}$ aproximadamente, el cual se divide en dos fuerzas de compresión $\mathrm{P}$, cada una de ellas en la dirección de las diagonales que concurren en el nudo, como se esquematiza en la Fig. 1 y que valen:

$$
P=E \cdot \cos 45^{\circ}=2.000 \times 0,707=1.414 \mathrm{kp}
$$

la cual a su vez se descompone en 4 partes, que si las espigas de la viga y sus cajas de largueros, es decir, si toda está bien construída a cada par de espigas o entalladuras le llega una fuerza de aprieto de:

$$
\frac{P}{4}=\frac{1.414}{4}=353 \mathrm{kp}
$$


como puede observarse en la Fig. 2, siendo pues la fuerza de armado de las espigas dos a dos, en este tipo de viga, del orden de $350 \mathrm{kp}$, pues el circuito de aire comprimido que suministra éste a los cilindros, bombines o gatos del banco de armado de vigas, varía entre dos límites: el mínimo para el cual el compresor se pone en marcha automáticamente, y el máximo de seguridad llegado el cual se detiene. Por eso como la presión del aire comprimido no es rigurosamente constante en cada momento, es por lo que decimos que esos $350 \mathrm{kp}$ es una fuerza de orden de esa magnitud, ya que por variar la presión del aire varía ella también. Pero las variaciones son pequeñas y se pueden despreciar.

\subsubsection{Resistencia del nudo sin encolar}

En la Fig. 2 se esquematiza cómo se distribuyen las distintas fuerzas que se originan al empujar cada espiga con esa fuerza hallada antes $P / 4 \approx 350 \mathrm{kp}$.

$$
\frac{\mathrm{P}}{4}=\mathrm{O}\left[\mu_{o}+\operatorname{tg}\left(\alpha+\mathrm{P}_{o}\right)\right] \quad \mathrm{Q}=\frac{\mathrm{P}}{4\left[\operatorname{tg} \varrho_{o}+\operatorname{tg}\left(\alpha+\varrho_{o}\right)\right]}
$$

y sustituyendo valores en (1):

$$
\mathrm{Q}=\frac{353}{4 \cdot \operatorname{tg}\left[\operatorname{tg} 18^{\circ}+\operatorname{tg}\left(6^{\circ} 30^{\prime}+18^{\circ}\right)\right]}=447 \mathrm{kp}
$$

luego la fuerza de arrancamiento necesaria por cada par de espigas asciende a:

$$
\begin{aligned}
& \frac{P^{\prime}}{4}=\mathrm{Q} \cdot\left[\operatorname{tg}\left(\alpha-\varrho_{o}\right)-\operatorname{tg} \varrho_{o}\right]=447 \cdot\left[\operatorname{tg}\left(6^{\circ} 30^{\prime}-18^{\circ}\right)-\operatorname{tg} 18^{\circ}\right]=-232 \mathrm{kp} \\
& P^{\prime}=-4 \times 232=-928 \mathrm{kp}
\end{aligned}
$$

es decir que, como cabía esperar, la fuerza necesaria de arrancamiento de una viga en celosía, bien construida y armada sin cola es una fuerza de tracción (obsérvese el cambio de signo de $P^{\prime}$ respecto a $P$ ), que por nudo vale menos que el empuje a que fue necesario llegar para armarlo, porque a su extracción ayudan las fuerzas $F$.

Queda pues comprobada la resistencia de los nudos de las vigas tipos STEIDLE-TREGAR, sin encolar, y su irreversibilidad, o imposibilidad de desarmado, mientras no se ejerzan tracciones en los nudos del orden de los $800 \mathrm{kp}$, cifra muy respetable en orden a la seguridad e inmobilización de la estructura de la viga.

\subsubsection{Presión de contacto}

Ya dijimos en el Epígrafe 1.5. que las espigas de las diagonales tienen entre sí una superficie de contacto de $23,45 \mathrm{~cm}^{2}$, y las espigas con las caras de las cajas labradas en los largueros o cordones de la viga que les sirven de alojamiento, de $40 \mathrm{~cm}^{2}$ Por tanto las presiones unitarias de contacto, siendo $Q=447 \mathrm{kp}$ la resultante de tales presiones en la pared de los alojamientos, y

$$
\mathrm{N}=\frac{\mathrm{Q}}{\cos \alpha}=\frac{447}{0,99357}=450 \mathrm{kp}
$$

la resultante de las presiones unitarias de las caras inclinadas de las espigas en contacto, serán: 


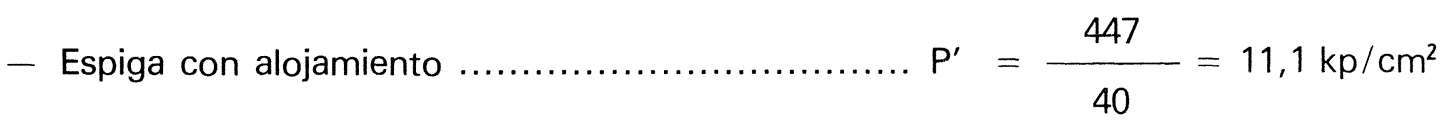

- Espiga con espiga .............................. $P^{\prime \prime}=\frac{450}{23,5}=19,1 \mathrm{kp} / \mathrm{cm}^{2}$

En el epígrafe 3.9. de la Parte $V$ publicada en el $n .^{\circ} 325$ de esta Revista, ya expusimos los tipos de colas que se fabrican en España para el encolado de este tipo de vigas. Pues bien: según especificaciones de los fabricantes en sus catálogos, para el Cascofen PA-8 es preciso que la presión se aplique uniformemente, lo que quiere decir que tanto las espigas como las caras internas de los alojamientos de los cordones donde aquéllas penetran deben estar correctamente labradas y en tal caso la presión óptima se determinará por ensayos, cuyos resultados dependerán de la compresibilidad de la madera y el estado de su superficie. Según el fabricante, a modo orientativo, para Obache y Calabó, la presión superficial basta con que sea de 7 a $8 \mathrm{kp} / \mathrm{cm}^{2}$, mientras que para Gaboon hacen falta de 10 a $12 \mathrm{kp} / \mathrm{cm}^{2}$ y para Makore es preciso llegar a los $14 \mathrm{kp} / \mathrm{cm}^{2}$.

En el caso que nos ocupa, la presión superficial oscila entre valores comprendidos de los límites dados por los autores citados, luego al mecanismo de armado y empuje de los cilindros o gatos neumáticos es correcta para la aplicación de una cola como la citada antes, a base de resina de fenol-formol, y mucho más aún para colas como el Cascofen RS-240-M o Aerodux RL 185 a base de resina de resorcina-formaldehído, que son las mejores resinas sintéticas conseguidas hasta el momento, por su contenido de resorcina, y que por tanto necesitan mínimas presiones superficiales de contacto. Pero además las dos presiones halladas cumplen perfectamente la norma DIN 68140 de octubre 1971 que da un limite mínimo de $10 \mathrm{kp} / \mathrm{cm}^{2}$.

\subsubsection{Características de la unión}

La entalladura o espiga de las vigas STEIDLE-TREGAR, que estamos analizando, tiene las siguientes características:

- Longitud

- Paso

- Grueso extremo de la entalladura

- Pendiente

- Ancho total de la unión

- Juego del fondo de entalladura

- Grado de debilitamiento: $\beta / P$

- Juego relativo entalladura: $S / L$

$$
\begin{aligned}
& \mathrm{L}=50 \mathrm{~mm} \\
& \mathrm{P}=10 \mathrm{~mm} \\
& \beta=2 \mathrm{~mm} \\
& \alpha=6^{\circ} 30^{\prime} \\
& \mathrm{H}=10 \mathrm{~mm} \\
& \mathrm{~S}=0 \\
& \mathrm{~V}=0,2 \mathrm{~mm} \\
& \mathrm{j}=0
\end{aligned}
$$

Por consiguiente, según Blömer, hace falta una superficie de contacto en el plano de cola $\mathrm{S}$ c con las características anteriores de:

$$
S_{c}=2 \cdot L(1-j)=10 \mathrm{~cm}^{2}
$$

y en el epigrafe 1.4., apartado 1.4.4., ya se dijo que la superficie de contacto entre espigas es de $23,5 \mathrm{~cm}^{2}$ y entre espiga y alojamiento de $40 \mathrm{~cm}^{2}$, por lo que por este lado la resistencia mecánica de los nudos de las vigas STEIDLE-TREGAR está asegurada.

En cuanto a la pendiente $\alpha=6^{\circ} 30^{\prime}$ es:

$$
\operatorname{tg} \alpha=0,11394=\frac{1}{8,777} \approx \frac{1}{9}
$$

y ya se sabe, como se explicó en la Parte VI publicada en el $n .^{\circ} 325$ de esta Revista que las pendientes óptimas, tanto desde el punto de vista de la resistencia de la unión, como 
de la del mecanizado de las entalladuras, oscilan entre $1 / 8$ y $1 / 10$, recordando también que en la Parte VI, apartado 3.1. se llega a la conclusión de que "a menor pendiente mayor resistencia a tracción de la unión por entalladura".

\subsection{Resistencia a torsión}

Las vigas de madera encolada para encofrados modernos están sometidas a duro trabajo y trato brutal, por parte del personal, que no es raro ver en obras que incluso están mal colocadas y arriostradas, cuando esto es necesario para evitar torceduras. Por ello es importante determinar cuál es su resistencia a tales torceduras en su plano, o sea el momento torsor que son capaces de resistir.

En el caso de la viga modelo STEIDLE-TREGAR que estamos estudiando, en la Fig. 3 se da su sección acotada, observándose que tal tipo de viga es, por su doble diagonal, una viga en cajón muy estrecha o alargada, para cuyo tipo de vigas, según S. Timoshenko, la tensión torsional máxima admisible vale aproximadamente:

$$
\tau_{\max }=\frac{\mathrm{M}_{t}}{\mathrm{~b} \cdot \mathrm{c}^{2}}\left(3+1,8 \frac{\mathrm{c}}{\mathrm{b}}\right)(2)
$$

en cuya expresión:

- $\tau_{\max }$ es la tensión torsional admisible máxima.

$-M_{t} \quad$ el momento torsor máximo admisible.

- b la longitud del mayor de los lados del cajón.

- c la longitud del menor de los lados del cajón.

Como $M_{t}$ da origen a unas $\tau$ rasantes, que de acuerdo con la norma DIN 1052, no deben pasar de $\tau_{\max }=8 \mathrm{kp} / \mathrm{cm}^{2}$, sustituyendo valores en (2) se deduce que este tipo de viga soporta admisiblemente un momento torsor de:

$$
M_{t} \leqslant 443 m \cdot k p
$$

despreciando los decimales, valor al que ni aproximadamente llegan los demás tipos de vigas que posteriormente estudiaremos. En realidad el valor real admisible es más bajo, porque las diagonales no forman una pared continua.

\subsection{Ensayos a rotura}

Para llevar a cabo el ensayo a rotura, se eligieron vigas al azar de las que entonces se estaban fabricando, que eran de $3,05 \mathrm{~m}$, y se remitieron al INSTITUTO EDUARDO TORROJA para su prueba a rotura, con el fin de determinar las cargas máximas de ruina, con las que calcular los momentos flectores y cortantes de rotura, y al compararlos con los admisibles, dados por el fabricante STEIDLE-TREGAR (español), calcular el coeficiente de seguridad y ver si es suficientemente amplio y del orden del calculado en el epigrafe 4 de la Parte IV publicada en el $n .^{\circ} 325$ de esta Revista.

En el Cuadro $n .^{\circ} 1$, se exponen los resultados del ensayo $n .^{\circ} 10.742-1$ hecho en el I.E.T.C.C. tomando como $\mathrm{M}_{a d m}$ el de 1.559 m.kp y no 1.500 estrictos para evitar interpolaciones. 
CUADRO N. ${ }^{\circ} 1$

\begin{tabular}{|c|c|c|c|c|c|c|}
\hline $\begin{array}{c}\text { VIGAS } \\
\text { N. }\end{array}$ & $\begin{array}{c}\text { VALOR DEL } \\
\text { MOVIMIENTO } \\
\text { FLECTOR } \\
\text { MEDIDO } \\
\mathrm{m} \cdot \mathrm{kp}\end{array}$ & $\begin{array}{c}\text { VALLOR DEL } \\
\text { FLEXIMETRO } \\
\text { EN } \\
\text { ELENTRO } \\
\mathrm{mm} \cdot 10^{-2}\end{array}$ & $\begin{array}{c}\text { FLECHA } \\
\text { EN EL } \\
\text { CENTRO } \\
\mathrm{mm}\end{array}$ & $\begin{array}{c}\text { LONGITUD } \\
\text { DE } \\
\text { VIGA } \\
\mathbf{m}\end{array}$ & $\begin{array}{c}\text { DISTANCIA } \\
\text { ENTRE } \\
\text { APOYOS } \\
\mathrm{m}\end{array}$ & $\begin{array}{c}\text { FLECHA RELATI- } \\
\text { VA RESPECTO A } \\
\text { DISTANCIA EN. } \\
\text { TRE APOYOS } \\
\mathrm{mm} / \mathrm{m}\end{array}$ \\
\hline 1 & 1.559 & 321 & 3,21 & 3,05 & 2,56 & 1,25 \\
2 & 1.559 & 310 & 3,10 & 3,05 & 2,56 & 1,21 \\
3 & 1.559 & 322 & 3,22 & 3,05 & 2,56 & 1,26 \\
4 & 1.559 & 316 & 3,16 & 3,05 & 2,56 & 1,23 \\
5 & 1.559 & 308 & 3,08 & 3,05 & 2,56 & 1,20 \\
\hline
\end{tabular}

La medida de las flechas obtenidas en condiciones óptimas de laboratorio es de 1,23 $\mathrm{mm} / \mathrm{m}$. Es lógico por tanto que el fabricante citado tome un margen de seguridad, para tener en cuenta las, a veces, brutales condiciones de trabajo en que se emplean las vigas, sin olvidar la perenne humedad. Por ello las fabrica con una flecha de $1,7 \mathrm{~mm} / \mathrm{m}$ que excede en un $38 \%$ al valor teórico medio obtenido por el INSTITUTO TORROJA.

\subsubsection{Resultados de los ensayos a rotura}

En el Cuadro n. ${ }^{\circ} 6$ del Informe del INSTITUTO TORROJA se dan resumidos los valores de los resultados de las 5 vigas ensayadas, de donde extraemos las siguientes medias:

\section{CUADRO N. ${ }^{\circ} 2$}

\begin{tabular}{|c|c|c|c|c|c|c|c|c|}
\hline \multirow[b]{2}{*}{$\begin{array}{l}\text { VIGAS } \\
\text { N. }^{\circ}\end{array}$} & \multirow{2}{*}{$\begin{array}{c}\text { LUZ } \\
\text { ENTRE } \\
\text { APOYOS } \\
m\end{array}$} & \multirow{2}{*}{$\begin{array}{c}\text { CARGA } \\
\text { TOTAL } \\
\text { DE } \\
\text { ROTURA } \\
\text { kp }\end{array}$} & \multicolumn{2}{|c|}{ REACCIONES } & \multirow{2}{*}{$\begin{array}{c}\text { MOMENTO } \\
\text { FLECTOR } \\
\text { MAXIMO } \\
\mathbf{m} \cdot \mathbf{k p}\end{array}$} & \multirow{2}{*}{$\begin{array}{c}\text { COEFIENTE } \\
\text { DE } \\
\text { SEGURIDAD } \\
\text { A MOMENTO } \\
\text { FLECTOR } \\
\mathrm{m}\end{array}$} & \multirow{2}{*}{$\begin{array}{c}\text { CORTANTE } \\
\text { MAXIMO } \\
\text { kp }\end{array}$} & \multirow{2}{*}{$\begin{array}{c}\text { COEFICIENTE } \\
\text { DE } \\
\text { SEGURIDAD } \\
\text { A CORTANTE } \\
\text { c }\end{array}$} \\
\hline & & & $\begin{array}{c}\text { EN A } \\
k p\end{array}$ & $\begin{array}{c}\text { EN B } \\
\text { kp }\end{array}$ & & & & \\
\hline MEDIA & 2,56 & 16.278 & $9.283,5$ & $|6.994,5|$ & 4.558 & 3 & 7.249 & 3,8 \\
\hline
\end{tabular}

\subsubsection{Coeficiente de seguridad}

Se han tomado como valores admisibles para el momento y el cortante, los dados por el fabricante de estas vigas en su catálogo, que son:

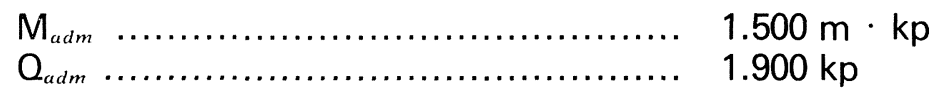

Téngase presente que casi siempre el esfuerzo predominante en estas vigas de madera encolada viene dado por el cortante, y es en él precisamente donde el coeficiente de seguridad es mayor, con buena diferencia sobre el que da el $\mathrm{M}_{u d m}$.

\subsubsection{Tensiones admisibles de trabajo}

Con el ensayo hecho por el INSTITUTO EDUARDO TORROJA, y los coeficientes de seguridad obtenidos a rotura, respecto de los valores admisibles de trabajo, quedan éstos comprobados pudiendo aceptarse con toda seguridad los dados por el fabricante, siempre, claro está, que las vigas estén bien fabricadas, como las ensayadas, elegidas al azar, y no haya una gran dispersión de resultados entre ellas, o sea, que sean lo más homogéneas posible.

Comprobemos ahora las tensiones admisibles del cuadro n. ${ }^{\circ} 6$ de la Norma DIN 1052 (epigrafe 1.1.), con las tensiones de trabajo obtenidas partiendo de la ficha técnica AITIM (epigrafe 1.3 de la Parte III publicada en el $\mathrm{n} .^{\circ} 324$ de esta Revista), y los coeficientes medios de seguridad determinados en el apartado 1.1. 
Tensión de rotura a flexión estática:

$\frac{850}{3}=283 \mathrm{kp} / \mathrm{cm}^{2}$

Tensión de compresión paralela a las fibras: $\frac{458}{3,8}=120 \mathrm{kp} / \mathrm{cm}^{2}$

Cortante a rotura paralelo a las fibras:

$\frac{115}{3,8}=30 \mathrm{kp} / \mathrm{cm}^{2}$

Tensión de compresión perpendicular a fibras: $\frac{91}{3,8}=24 \mathrm{kp} / \mathrm{cm}^{2}$

Cortante tangencial de las fibras:

$\frac{20}{3,8}=5 \mathrm{kp} / \mathrm{cm}^{2}$

\section{Vigas de celosia tipo Warren con diagonal única}

Vigas de madera encolada para encofrados conocemos la marca PERI que vamos a analizar, comenzando por hacer notar que los valores característicos que da el fabricante en sus catálogos como admisibles inducen a error, pues resalta los máximos admisibles posibles y minimiza los mínimos, que son precisamente los que hay que tener en cuenta en un estudio analítico como el presente.

En el Cuadro $n .^{\circ} 3$ de la Parte I ya quedaron consignados, y son:

$$
\begin{aligned}
& \mathrm{M}_{\text {adm }}=1.600 \mathrm{~m} \cdot \mathrm{kp} \\
& \mathrm{Q}_{\text {adm }}=1.650 \mathrm{kp}
\end{aligned}
$$

que referidos al peso unitario de este tipo de viga, $7,8 \mathrm{kp} / \mathrm{m}$, más elevado que la modelo STEIDLE-TREGAR, porque sus cordones tienen un mayor canto, $70 \mathrm{~mm}$, contra $60 \mathrm{~mm}$ en la última citada, dan unas características específicas, consignadas en dicho Cuadro n. ${ }^{\circ} 3$ de la Parte I, que son inferiores a las ofrecidas por la viga del modelo estudiado antes, 0 sea:

$$
\begin{aligned}
& M_{\text {especifico }}=\frac{1.600}{7,8}=205 \\
& \mathrm{Q}_{\text {especifico }}=\frac{1.650}{7,8}=211
\end{aligned}
$$

\subsection{Cordón superior}

La altura o canto de la viga también es de $360 \mathrm{~mm}$, medida a exteriores de cordones, y su escuadría es de $70 \times 100 \mathrm{~mm}$, de manera que la distancia vertical, o entre centros de gravedad de cordones, es de $290 \mathrm{~mm}$, luego la carga máxima admisible a que se pueden someter los cordones es:

$$
\frac{1.600}{29} \times 100= \pm 5.517 \mathrm{kp}
$$

que en el caso del cordón superior será de compresión, adoptando para ese sentido del esfuerzo o carga al signo negativo.

Aquí también es válido el argumento expuesto en el apartado (1.1.), al definir la longitud de cálculo a pandeo del cordón superior, y como la celosía es tipo Warren formando las diagonales ángulo recto entre sí, la distancia entre ejes de nudos será también de $640 \mathrm{~mm}$. 
El área de la sección transversal de los cordones vale:

$$
A=10 \times 7=70 \mathrm{~cm}^{2}
$$

y su mínimo módulo resistente:

$$
W_{\text {min }}=\frac{1}{6} \times 10 \times 7^{2}=82 \mathrm{~cm}^{3}
$$

despreciando los decimales por defecto inferiores a 0,5 inclusive.

El momento de inercia de tales cordones asciende a:

$$
I_{\text {min }}=\frac{1}{12} \times 10 \times 7^{3}=286 \mathrm{~cm}^{4}
$$

luego el radio de giro de tal sección transversal será:

$$
i=\sqrt{\frac{286}{70}}=2 \mathrm{~cm}
$$

y la esbeltez del cordón superior entre nudos:

$$
\lambda=\frac{32}{2}=16
$$

para la cual corresponde un coeficiente de pandeo, según DIN 1052 de:

$$
\omega=1,06
$$

Por lo que se refiere al momento flector, para la luz límite media de $3,6 \mathrm{~m}$, la carga uniformemente repartida a lo largo de la viga será de:

$$
\mathrm{q}_{a d m}=\frac{2 \times 1.650}{3,6}=917 \mathrm{kp} / \mathrm{m}
$$

$y$ el momento de empotramiento en el comienzo del nudo vale:

$$
M=105 \times 9,17=963 \mathrm{~cm} \cdot \mathrm{kp}
$$

luego la tensión máxima será:

$$
\sigma=\frac{5.517 \times 1,06}{70}+0,86 \times \frac{963}{82}=93,5 \mathrm{kp} / \mathrm{cm}^{2}<\sigma_{c_{a d m}}
$$

\subsection{Cordón inferior}

Siendo la carga de $+5.517 \mathrm{kp}$, o sea de tracción, según el convenio de signos adoptado, la tensión de trabajo será:

$$
\sigma=\frac{5.517}{70}=79 \mathrm{kp} / \mathrm{cm}^{2}<\sigma_{t_{a d m}}
$$

\subsection{Diagonal comprimida}

Las diagonales de este modelo de viga se componen de un solo elemento de escuadria maciza de $60 \times 60 \mathrm{~mm}$, y la distancia entre ejes de cordones es de $290 \mathrm{~mm}$, de manera que la longitud de las diagonales entre ejes de cordones es:

$$
290 \times \sqrt{2}=410 \mathrm{~mm}
$$


y sus características mecánicas son:

$$
\begin{aligned}
A & =6 \times 6=36 \mathrm{~cm}^{2} \\
\mathrm{I}_{\text {min }} & =\frac{1}{12} \times 6 \times 6^{3}=108 \mathrm{~cm}^{4} \\
\mathrm{~W}_{\text {min }} & =\frac{1}{6} \times 6 \times 6^{2}=36 \mathrm{~cm}^{3} \\
i & =\sqrt{\frac{108}{36}}=1,73 \mathrm{~cm}
\end{aligned}
$$

En cuanto a la luz de cálculo a pandeo, tomando la longitud libre entre caras interiores de cordones será:

$$
l=220 \times \sqrt{2}=311 \mathrm{~mm} \approx 31 \mathrm{~cm}
$$

luego la esbeltez valdrá:

$$
\lambda=\frac{31}{1,73}=17,9 \sim 18
$$

para lo cual:

$$
\omega=1,07
$$

Evidentemente que la diagonal más cargada será una de las que arranquen de los apoyos, como ya comprobamos en el caso anterior del epígrafe 1. y como el fabricante de estas vigas da como valor mínimo del esfuerzo cortante, el ya indicado de $1.650 \mathrm{kp}$, que lógicamente por norma será el admisible, y un máximo de $2.600 \mathrm{kp}$, la diagonal comprimida más cargada trabajará entre los siguientes límites:

$$
\begin{aligned}
& 1.650: \cos 45^{\circ}=2.333 \mathrm{kp}_{\min } ; 2.600: \cos 45^{\circ}=3.677 \mathrm{kp}_{\max } \\
& \sigma_{c}=\frac{2.333 \times 1,07}{36}=69,3 \mathrm{kp} / \mathrm{cm}^{2}<\sigma_{c_{a d m}}^{\prime \prime} \text {; } \\
& \sigma_{c}=\frac{3.677 \times 1,07}{36}=109 \mathrm{kp} / \mathrm{cm}^{2}>\sigma_{c_{a d m}}
\end{aligned}
$$

luego este fabricante es un poco optimista a la hora de redactar sus catálogos, y el usuario debe poner precaución de su parte para usar sus vigas con todos los valores de cargas que para ellas da.

\subsection{Diagonales extendidas}

Es evidente que si el fabricante de las vigas de madera encolada en celosía tipo Warren, marca PERI, da para el valor de Q cifras que oscilan entre $1.650 \mathrm{kp}$ y $2.600 \mathrm{kp}$, la diagonal extendida más cargada lo será con un valor igual, pero de signo contrario a los determinados antes para la más comprimida, porque este modelo de vigas PERI no termina en uno de sus extremos en voladizo, sino que ambos son iguales y terminan en montante recto.

Asi pues la diagonal más cargada a tracción sufrirá las siguientes tensiones:

$$
\sigma_{\text {min }}=\frac{2.333}{36}=65 \mathrm{kp} / \mathrm{cm}^{2}<\sigma_{t_{a d m}} ; \sigma_{\max }=\frac{3.677}{36}=102 \mathrm{kp} / \mathrm{cm}^{2}>\sigma_{t_{a d m}}
$$

resultados que corroboran lo dicho respecto del optimismo del catálogo de PERI. 


\subsection{Cálculo de los nudos.}

En el caso de la viga PERI los nudos están formados por espigas o entalladuras cortas o minientalladuras (ver Fig. 2 de la Parte I) que según la norma DIN 68140 de junio 1960, pertenecen al grupo de esfuerzo II, y su empleo está previsto para "ventanas, puertas, suelos, mobiliario, etc., pero no para piezas de construcción que se tengan que calcular con arreglo a la norma DIN 1052, o que se exponen a esfuerzos mecánicos muy altos".

Su corta longitud de entalla, de 7 a $10 \mathrm{~mm}$ (ver Fig. 2 de la Parte l) exige prensas de armado fuertes y costosas, capaces de alcanzar la presión de armado de la viga adecuada a esa corta longitud (véase Gráfico 1 de la Parte VI).

El ángulo de las 'entallas es 2. $\alpha=20^{\circ}$, y la superficie de contacto entre caras de cuñas es de $5 \mathrm{~cm}^{2}$ y cada diagonal tiene 10 entallas de $100 \mathrm{~mm}$ de longitud horizontal, que se introducen en cuatro cajas, labradas en los largueros o cordones de $10 \mathrm{~mm}$ de ancho.

\subsubsection{Coeficiente de rozamiento.}

Nada hay que añadir a lo dicho en el apartado 1.4.1, puesto que la madera con que están construidas todas las vigas de madera encolada para encofrados modernos es de calidad similar, así que $\mu_{o}$ (en reposo) $=0,34$, lo que da tg $\varrho_{o}=0,34$, o sea $\varrho_{o}=18^{\circ} 46^{\prime}$, pero adoptamos $Q_{o}=18^{\circ}$ para mayor seguridad en los sucesivos cálculos, luego $\operatorname{tg} \sigma_{o}=\mu_{o}=$ $=0,32491$.

\subsubsection{Fuerza de armado.}

Como desconocemos las características de los bombines neumáticos empleados por el fabricante de las vigas marca PERI, que estamos estudiando, supondremos, en primera aproximación, que tales gatos o bombines son iguales a los utilizados por el fabricante español de las vigas STEIDLE-TREGAR, recordando lo dicho en el apartado 1.5.2., que el empuje $E=2.000 \mathrm{kp}$, y como las diagonales forman una celosia con un ángulo entre sí de $90^{\circ}$, a cada diagonal le llegarán, como en ese apartado 1.4.2., se calculaba, $1.414 \mathrm{kp}$.

Como ya hemos dicho en el apartado 2.5. que cada diagonal de viga PERI tiene 10 entalladuras, esos $1.414 \mathrm{kp}$ se tendrán que repartir lo más uniformemente posible entre ellos, tocando a cada una $141,4 \mathrm{kp}$ que redondeando

$$
\frac{P}{10}=140 \mathrm{kp}
$$

por la dificultad del reparto uniforme de $\mathrm{P}$ entre las 10 entallas.

\subsubsection{Resistencia del nudo sin encolar.}

En las Figs. 1 y 2 se esquematizan cómo se distribuyen las fuerzas que se originan al empujar cada espiga, cuña o entalla con la fuerza antes supuesta $P / 10 \approx 140 \mathrm{kp}$.

La cuña de la viga PERI es irreversible por cuanto:

$$
2 \cdot \alpha=20^{\circ}<2 \cdot \varrho_{o}=36^{\circ}
$$

pero por ser cuña doble, requiere una fuerza de penetración doble también que la cuña sencilla, como la de la viga STEIDLE-TREGAR.

La fuerza de penetración vale:

$$
\frac{\mathrm{P}}{10}=2 \cdot \mathrm{Q} \cdot \operatorname{tg}\left(\alpha+\varrho_{0}\right)
$$




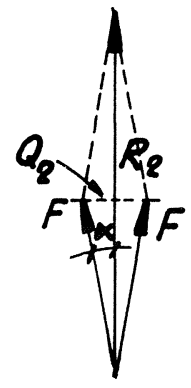

Fig. 4

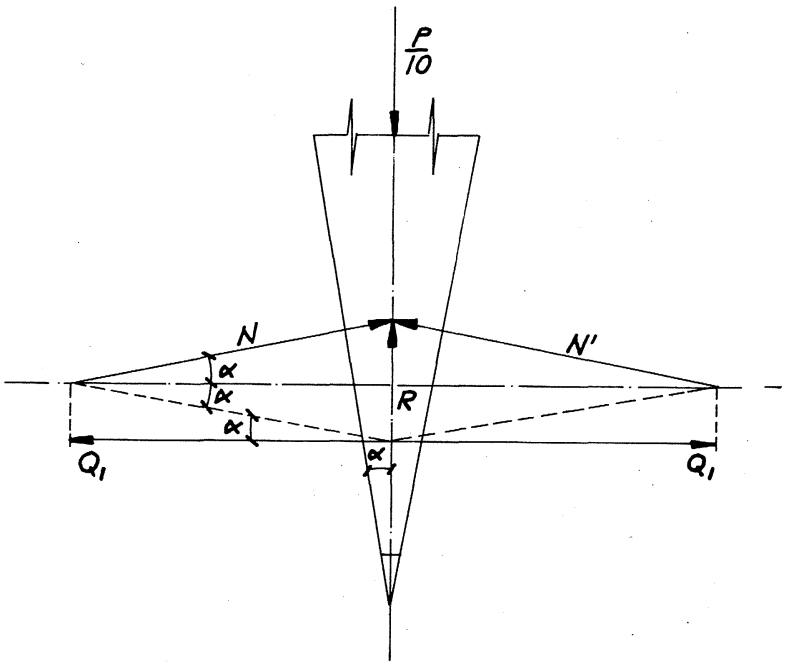

Fig. 5

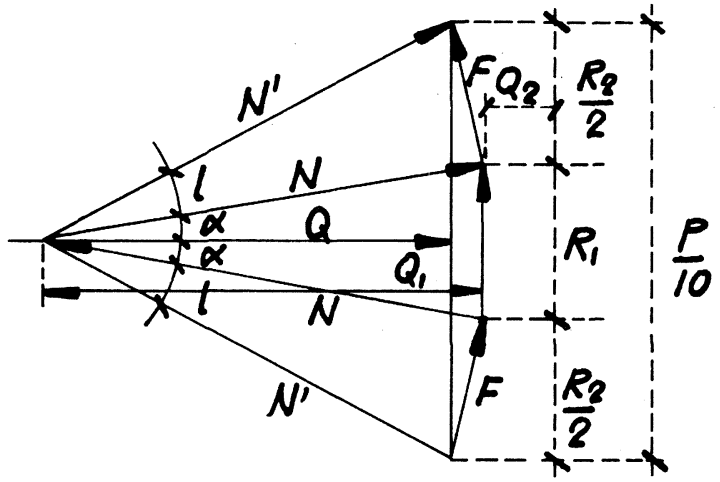

Fig. 6

contra los $447 \mathrm{kp}$ que se calcularon para la viga STEIDLE-TREGAR en el apartado 1.4.3.

Si intentamos sacar c extraer las diagonales de la viga PERI, en el supuesto del aprieto considerado, se puede demostrar que la fórmula que nos da $P^{\prime}$ es:

$$
\mathrm{P}^{\prime}=2 \cdot \mathrm{Q} \cdot \operatorname{tg}\left(\alpha-\varrho_{o}\right)
$$

y sustituyendo valores llegamos al valor de - P', que es:

$$
\mathrm{P}^{\prime}=2 \times 165 \cdot \operatorname{tg}\left(10^{\circ}-18^{\circ}\right)=-46 \mathrm{kp}
$$

contra los - $928 \mathrm{kp}$ necesarios para desarmar los nudos de la viga STEIDLE-TREGAR (véase apartado 1.5.3.).

Estos modestos $46 \mathrm{kp}$ casi permiten desarmar a mano la viga PERI, sin encolar, a no ser, como ya lo hemos anunciado antes, que este fabricante disponga de banco de armado, provisto de gato o bombines y presión neumática, mucho mayores que los necesarios para armar la viga STEIDLE-TREGAR, cosa casi segura pues el problema fundamental de las entalladuras de la viga PERI, es que tienen un ángulo $2 \cdot \alpha$ muy próximo al doble del ángulo de rozamiento $2 \cdot \varrho_{o}$ es decir, que están muy cerca del límite de la irreversibilidad de la cuña, lo que es un evidente peligro para el empleo de estas vigas, en las que, como hemos visto, la resistencia de los nudos sin encolar es muy pequeña, y aquélla hay que confiarla casi totalmente al encolamiento y calidad de la cola, y lo primero es difícilmente ejecutable con uniformidad de garantía, pues el encolamiento se realiza manualmente.

Por ello es seguro que el fabricante de las vigas PERI, se ha tenido que ver obligado a proveerse de imponente banco de montaje, y a emplear presiones neumáticas elevadas, con la consiguiente repercusión en la inversión en gatos y montaje de vigas. 


\subsubsection{Presiones de contacto.}

Las fuerzas de contacto entre las caras de las entalladuras que se tocan valen:

$$
\begin{gathered}
\mathrm{Q}^{\prime}=\frac{\mathrm{Q}}{1-\operatorname{tg} \alpha \cdot \operatorname{tg} \varrho_{o}}=\frac{165}{1-0,17632 \times 0,32491}=175 \mathrm{kp} \\
\mathrm{N}=\frac{\mathrm{Q}^{\prime}}{\cos \alpha}=\frac{175}{0,9848}=178 \mathrm{kp}
\end{gathered}
$$

de modo que las presiones de contacto entre caras de espigas o entalladuras, y de las espigas con su alojamiento en las cajas labradas en los largueros o cordones, que supondremos se tocan los mismos $40 \mathrm{~cm}^{2}$ que dimos en el apartado 1.5.4., dada la similitud geométrica de las celosías de las vigas marca STEIDLE-TREGAR y PERI, serán:

$$
\begin{aligned}
& \text { - Espiga con alojamiento ....... } \mathrm{p}^{\prime}=\frac{175}{40}=4,4 \mathrm{kp} / \mathrm{cm}^{2}<\text { mín. } \\
& \text { - Espiga con espiga ............ } \mathrm{p}^{\prime \prime}=\frac{178}{5}=35,6 \mathrm{kp} / \mathrm{cm}^{2}>\text { mín. }
\end{aligned}
$$

siendo, como se dijo en el apartado 1.5.4., la presión mínima admitida por la norma DIN 68140 de octubre de $1971.10 \mathrm{kp} / \mathrm{cm}^{2}$, luego nuevamente comprobamos que la resistencia del nudo de las vigas PERI, está confiada al encolamiento, o su fuerza de armado, que hemos supuesto con un empuje $E=2.000 \mathrm{kp}$ por cada gato 0 bombin, que actúa sobre cada nudo de la celosía, no puede ser inferior a 2,3 veces, o sea a $4.600 \mathrm{kp}$, para que p' antes calculada, no sea menor de los $10 \mathrm{kp} / \mathrm{cm}^{2}$ especificados en la citada norma.

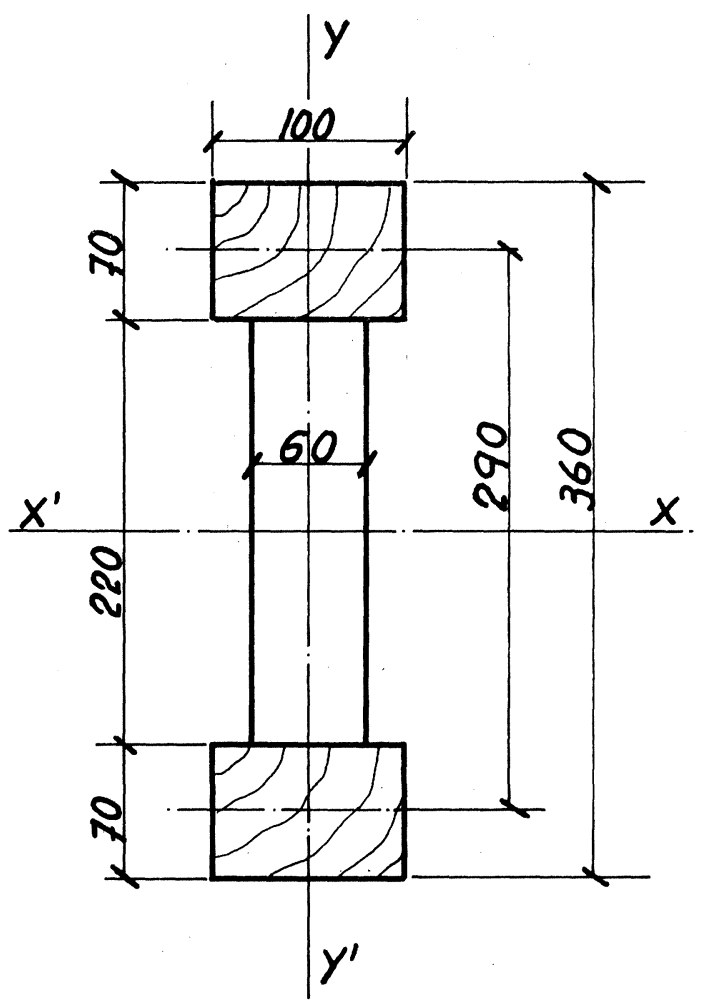

Fig. 7

\subsubsection{Características de la unión}

Las características de las minientalladuras de los nudos de la celosía de las vigas marca PERI son:

- Longitud $\mathrm{L}=10 \mathrm{~mm}$

- Paso $P=3,6 \mathrm{~mm}$

- Grueso extremo entalladura ....... $\beta=0 \mathrm{~mm}$

- Pendiente ............................. $\quad \alpha=10^{\circ}$

- Ancho total de la unión ........... H $=9 \mathrm{~mm}$

- Juego fondo entalladura ............ $S=0$

- Grado de debilitamiento: $\beta / P$..... $V=0$

- Juego relativo entalladura: $S / L . . \quad j=0$

Según Blömer hace falta una superficie de contacto en el plano de la cola $S_{c}$, con las características anteriores de

$$
\mathrm{S}_{c}=2 \cdot L \cdot(1-\mathrm{j})=2 \mathrm{~cm}^{2}
$$

$y$ en realidad existen en la viga estudiada $5 \mathrm{~cm}^{2}$, por lo que cumple esta condición.

En cuanto a la pendiente $\alpha=10^{\circ}$ es:

$$
\operatorname{tg} \alpha=0,17632=\frac{1}{5.6715} \approx \frac{1}{6}
$$


valor que está alejado de las pendientes óptimas dadas en la Parte VI, que deben oscilar entre $1 / 8$ y $1 / 10$, recordando que en dicha Parte VI, ya se explicó que "a menor pendiente mayor resistencia a tracción de la unión por entalladuran, y al contrario, como ocurre con la unión entallada de las vigas marca PERI, pues $1 / 6$ es un $50 \%$ superior a la media de los limites antes citados,

\subsection{Resistencia a torsión}

La viga modelo PERI posee diagonales de una sola pieza de escuadria $60 \times 60 \mathrm{~mm}$ (véase la Fig. 7), luego su sección transversal no presenta forma de viga en cajón, por lo que según Timoshenko, en su libro "Resistencia de Materiales", tomo II, pág. 290, para este tipo de vigas el momento flector máximo en el ala es aproximadamente triple del momento torsor $M_{1}$, con tal de que la viga sea lo suficientemente larga para que $T h(\ell / a)$ tienda a 1. Por ejemplo si $\ell /$ a y $\ell \geqslant 6 \cdot h=6 \times 36=216 \mathrm{~cm}$ (prácticamente las vigas de madera encolada en celosía para encofrados se usan a partir de la $3,05 \mathrm{~m})$, entonces Th $(\ell / \mathrm{a})=$ $=0,96$ y el error que se comete es de sólo un $4 \%$.

Pues bien: como el fabricante de la viga PERI da para ella un $\mathrm{M}_{t_{\text {adm }}}=1.600 \mathrm{~m} \cdot \mathrm{kp}$, para no rebasar en torsión las tensiones admisibles, según Timoshenko.

$$
\mathrm{M}_{i}=\frac{\tau_{a d m}}{\sigma_{f_{\text {adm }}}} \cdot \frac{\mathrm{M}_{f}}{3}=\frac{8}{124} \times \frac{1.600}{3}=34 \mathrm{~m} \cdot \mathrm{kp}
$$

valor inferior al admisible para la viga STEIDLE-TREGAR que es $41,3 \mathrm{~m} \cdot \mathrm{kp}$, lo que representa un $17,7 \%$ de menor resistencia a torsión.

\section{Vigas doble $\mathrm{T}$ de alma llena}

En el mercado de vigas de madera encolada para encofrados modernos, existe un tipo de doble T marca DOKA, cuya perspectiva se da en la Fig. 8, que el autor juzga suficientemente clara como para evitar su descripción.

De esta marca DOKA existen, que sepa el autor, cuatro modelos en el mercado: el $\mathrm{H} 20$ de $200 \mathrm{~mm}$ de altura; el H 30 de $300 \mathrm{~mm}$ de altura total; el H 36 de $360 \mathrm{~mm}$ de altura total y el H 36, de $360 \mathrm{~mm}$ de altura total, pero que uno de sus extremos no termina en sección perpendicular al eje longitudinal de la viga, sino que acaba con una sección que forma un ángulo de $45^{\circ}$ con dicho eje.

En las Figs. 9, 10 y 11 se dan las dimensiones de las secciones transversales de los modelos $\mathrm{H} 20 ; \mathrm{H} 30$ y $\mathrm{H} 36$ respectivamente.

Como las vigas estudiadas en los epígrafes anteriores, 1. y 2., así como en el próximo 4., todas tienen una altura o canto de $360 \mathrm{~mm}$, de los tres o mejor cuatro modelos que tiene en el mercado la viga DOKA elegimos el $\mathrm{H} 36$, de 360 de altura o canto, para la cual el fabricante da en sus catálogos las caracteristicas siguientes:

$$
\begin{aligned}
& \mathrm{M}_{\text {adm }}=1.700 \mathrm{~m} \cdot \mathrm{kp} \\
& \mathrm{Q}_{a d m}=1.600 \mathrm{kp}
\end{aligned}
$$

y repasando el Cuadro $n .{ }^{\circ} 3$ de la Parte I, puesto que esta viga pesa $9 \mathrm{kp} / \mathrm{m}$, sus valores específicos serán:

$$
\begin{aligned}
& M_{\text {especifico adm }}=189 \\
& Q_{\text {especifico adm }}=178
\end{aligned}
$$

valores sensiblemente inferiores a los que dan las vigas en celosía, lógicamente más ligeras. 


\subsection{Cordón superior}

Siendo $307 \mathrm{~mm}$ la distancia vertical entre ejes de cordones, éstos estarán sometidos a unos esfuerzos iguales y contrarios de:

$$
M=\frac{1.700 \times 100}{30,7}= \pm 5.537 \mathrm{kp}
$$

en el supuesto de que la unión entallada entre alma y cordones no tenga deslizamiento, cosa que comprobaremos más adelante, por tanto en este primer supuesto las caracteristicas mecánicas de la sección de la viga DOKA H 36 son: (Véase Cuadro n. ${ }^{\circ} 3$ de la Parte Parte I).

$$
\begin{aligned}
I_{x x \prime} & =29.942 \mathrm{~cm}^{4} \\
W_{x x,} & =\frac{29.942}{36: 2}=1.663 \mathrm{~cm}^{3} \\
\sigma_{f} & =\frac{1.700 \times 100}{1.663}= \pm 102 \mathrm{kp} / \mathrm{cm}^{2}<\sigma_{f_{a d m}}
\end{aligned}
$$

luego se observa que el catálogo de DOKA, al asegurar a su viga modelo $\mathrm{H} 36$ un momento flector admisible de $1.700 \mathrm{~m} \cdot \mathrm{kp}$, que como vemos produce unas tensiones máximas de flexión de $\pm 102 \mathrm{kp} / \mathrm{cm}^{2}$, cuando la máxima admisible (véase epigrafe 1.1.) es de \pm 124 $\mathrm{kp} / \mathrm{cm}^{2}$, según la norma DIN 1052 después de minorada por la humedad y mayorada por la hipótesis de cálculo $\mathrm{HZ}$, cumple dicha norma.

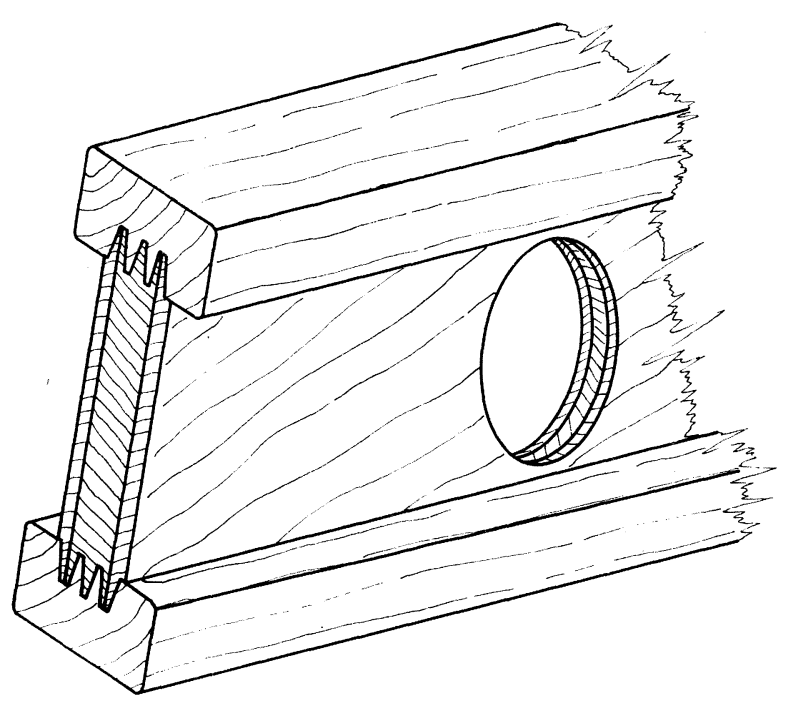

Fig. 8

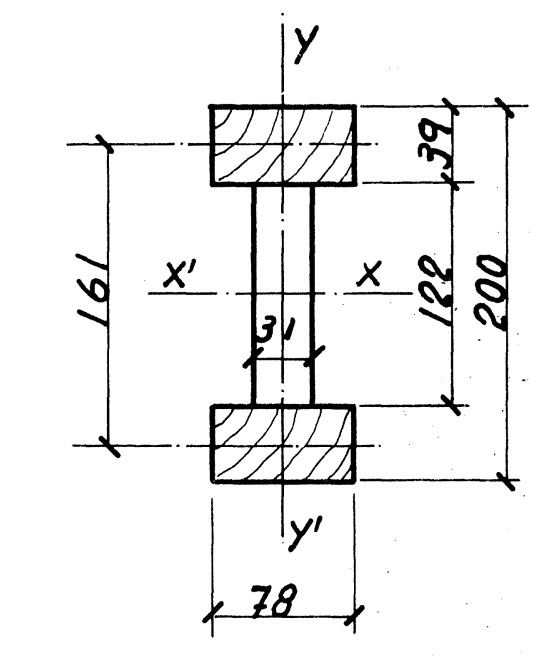

Cotas en mm

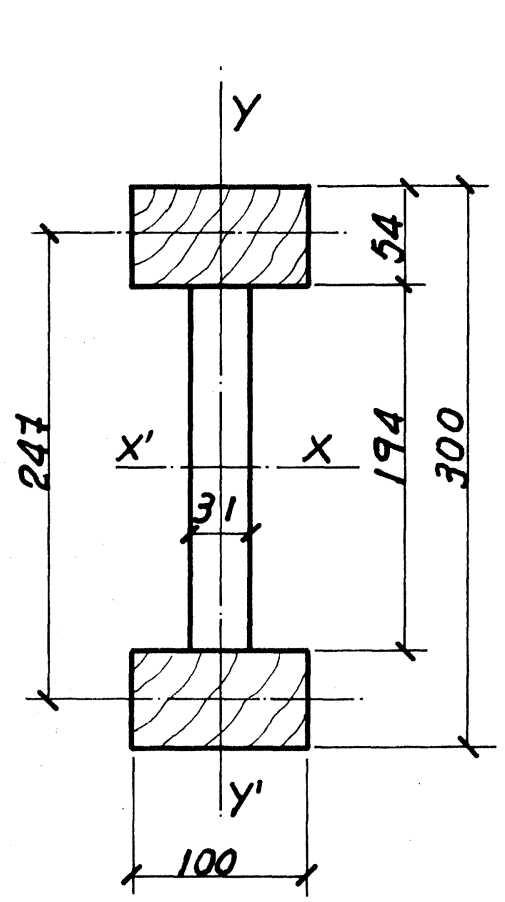

Fig. 10

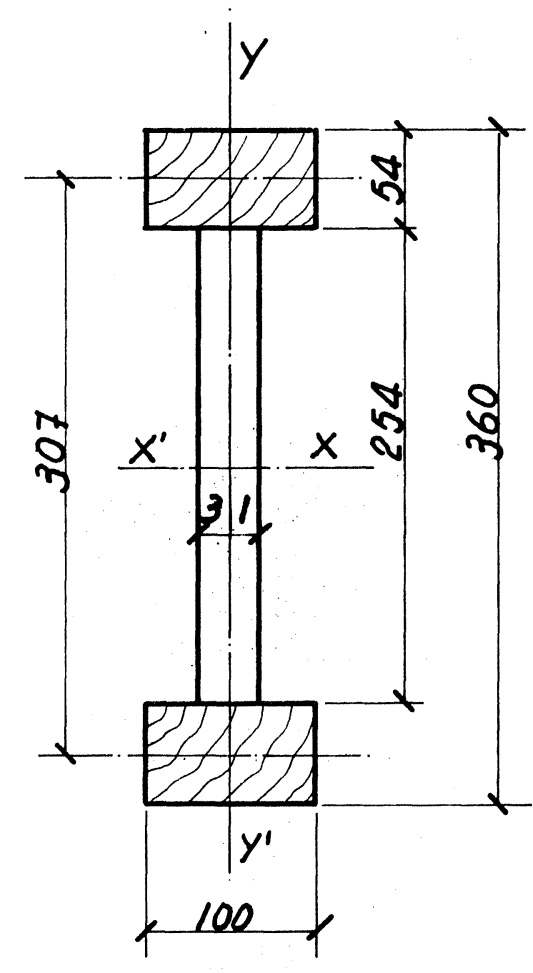

Fig. 11 


\subsection{Cordón inferior}

Poco hay que decir sobre este pues, supuesta la simetría perfecta de la viga, como se han dibujado en las Figs. 9, 10 y 11, la máxima tensión a flexión será también la hallada antes, o sea $\pm 102 \mathrm{kp} / \mathrm{cm}^{2} \sigma_{i_{\text {udm }}}$. según DIN 1052 .

\subsection{Alma}

Como se sabe por "Resistencia de Materiales", prácticamente en las vigas doble T las alas no colaboran para resistir el cortante que dichas vigas tienen que soportar en apoyos, normalmente donde, como ocurre con las vigas de madera para encofrados modernos, se presentan las cargas mayores y el momento flector es nulo, y por tanto máximo el cortante.

Se sabe también que la tensión cortante máxima vale:

$$
\tau_{\max }=\frac{C \cdot \boldsymbol{m}}{1 \cdot b}
$$

en cuya expresión:

C es el cortante máximo

$\mathcal{M}$ es el momento estático de la sección de la viga

I es el momento de inercia de la viga

b es el ancho del alma.

Es fácil demostrar que $\tau_{\max }$ se presenta en la línea neutra en las vigas metálicas doble $T$ de alma llena, y operando se llega a la siguiente fórmula simplificada:

$$
\tau_{\max }=1,5 \cdot \frac{\mathrm{C}}{\mathrm{b} \cdot \mathrm{h}}
$$

en la que $\mathrm{C}$ es el máximo cortante; $\mathrm{b}$ el ancho del alma y $\mathrm{h}$ la altura de ésta.

Pero esta fórmula es válida para el material que hemos indicado, pues el Laboratorio de Productos Forestales Americanos, ha llevado a cabo exhaustivos ensayos de los que se deduce que cuando la carga está próxima al apoyo, la distribución no es como en el centro de la viga, y que gracias a que la sección del alma se comporta como si estuviera formada por dos mitades independientes, se obtiene cierta mejora en el valor del cortante en el eje neutro. Ver Figs. 12, 13 y 14.

De la inspección de tales figuras se observa que los esfuerzos cortantes tienden, conforme se acercan al apoyo, a disminuir en el eje neutro y a desplazarse hacia los bordes, es decir, en el caso de la viga DOKA, hacia la unión entallada del alma con los cordones, que es el punto débil de este tipo de viga, como veremos.

Por de pronto comprobaremos el alma, que tiene una sección de $3,1 \times 25,4=79 \mathrm{~cm}^{2}$. 
Suponiendo la viga de luz límite, que calculamos en el epigrafe 5 de la Parte I, que es de $3,6 \mathrm{~m}$, longitud que está prevista en el catálogo DOKA,

$$
\begin{gathered}
\mathrm{M}_{\text {alm }}=1.700=\frac{\mathrm{p} \cdot 3,6^{2}}{8} ; \mathrm{p}=1.049 \mathrm{kp} / \mathrm{m} \\
\mathrm{C}=\mathrm{R}_{A}=\mathrm{R}_{B}=\frac{1.049 \times 3,6}{2}=1.888 \mathrm{kp} \\
\tau_{\text {max }}=1,5 \cdot \frac{1.888}{3,1 \times 25,4}=36 \mathrm{kp} / \mathrm{cm}^{2}
\end{gathered}
$$

y como hay tres cuñas a dos caras, hay que dividir por 6 , asi que $\tau_{\max }=6 \mathrm{kp} / \mathrm{cm}^{2}$, y en el epígrafe 1.1. calculamos las tensiones admisibles según DIN 1052, minoradas por humedad y mayoradas por tener en cuenta el caso de carga' $\mathrm{HZ}$ y dieron a cortadura $\tau_{u d m}=$ $=8 \mathrm{kp} / \mathrm{cm}^{2}$.

Pero en la viga DOKA se observa una gran anormalidad en el reparto de esa tensión rasante $\tau$, como lo muestran las Figs. 12, 13 y 14.
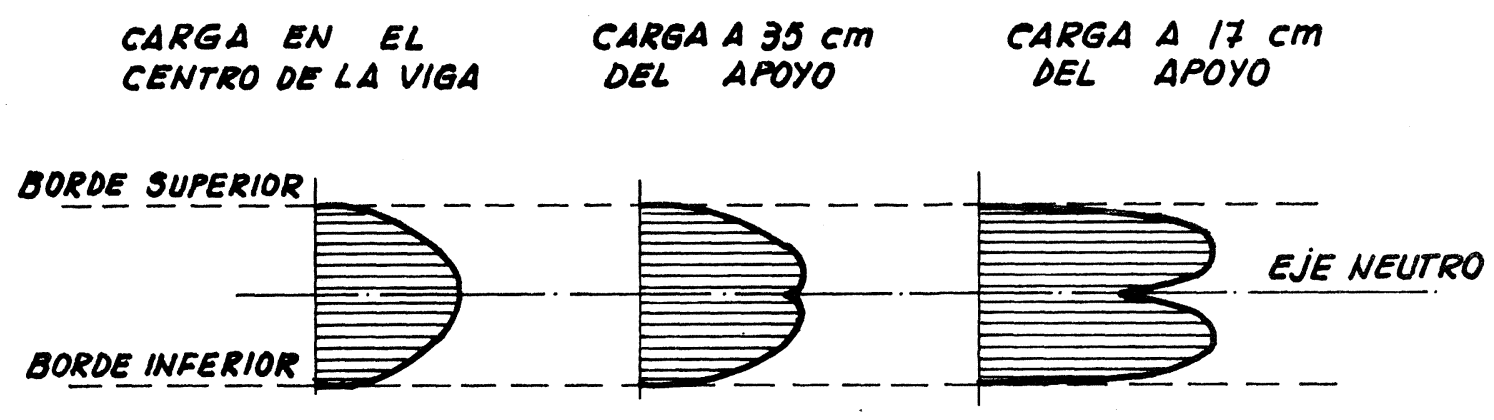

Fig. 12

Fig. 13

Fig. 14

De todas formas vamos a ser más precisos en la comprobación del alma, sabiendo que está formada por tres tableros contrachapados, cuyas fibras son perpendiculares en los adyacentes.

La norma DIN 1052 da, en su Cuadro n. ${ }^{\circ}$ 8, las tensiones admisibles de cortadura para placas de enchapado, que son: $9 \mathrm{kp} / \mathrm{cm}^{2}$ para cortantes en el plano de las placas, y $18 \mathrm{kp} / \mathrm{cm}^{2}$ en cortantes perpendiculares al plano de las placas. Luego tomando este valor último, y corrigiéndolo con la minoración de humedad y mayoración de tipo de carga $\mathrm{HZ}$ tendremos una tensión admisible de:

$$
\tau_{u d m}=18 \times \frac{5}{6}+15 \%=17 \mathrm{kp} / \mathrm{cm}^{2}
$$

que es la mitad de la obtenida con el $\mathrm{M}_{i_{u d m}}=1.700 \mathrm{~m} \cdot \mathrm{kp}$ y la luz límite de 3,6 $\mathrm{m}$.

Hagamos una tercera comprobación: El catálogo DOKA da para su viga aquí estudiada H $36 \mathrm{~m}$ cortante admisible de $1.600 \mathrm{kp}$. Tomando esta cifra experimental del fabricante:

$$
\tau_{\max }=1,5 \times \frac{1.600}{3,1 \times 25,4}=30 \mathrm{kp} / \mathrm{cm}^{2}
$$


la cual hay que dividirla por 6 , quedando $\tau_{\max }=5 \mathrm{kp} / \mathrm{cm}^{2}<\tau_{\text {adm }}$ en el supuesto de reparto uniforme de que se está muy alejado y ello explica, junto con los cálculos que seguirán, que ese punto débil de esta viga produce su envejecimiento prematuro por roturas, alabeos, despegues de las capas, etc., de su alma, accidentes estos comprobados en Alemania.

Otra anormalidad importante en las vigas DOKA es la ausencia de rigidizadores. Siguiendo al Catedrático, don Ramón Argüelles Alvarez, en su obra "Cálculo de Estructuras de Maderan, diremos que las costillas de rigidez que se disponen en las vigas doble $T \circ$ en cajón, tienen la misma justificación que en las vigas metálicas armadas. Su función es: evitar el pandeo de las almas de las vigas; mantener fija la posición relativa de las cabezas o cordones; distribuir las cargas concentradas, y facilitar la construcción de la jácena al formar un esqueleto rígido.

Desde el punto de vista del proyecto, se clasifican en dos grupos: los que distribuyen cargas concentradas fuertes - tales como los que se disponen en los apoyos-, y los que estabilizan el alma de la viga. Naturalmente algunos rigidizadores satisfacen ambos propósitos.

El emplazamiento de los rigidizadores que reparten las cargas concentradas viene aplicado por las condiciones exteriores, es decir, por la situación de los apoyos, y por la ubicación de dichas cargas, Fig. 15. Las dimensiones de estos rigidizadores se deducen dividiendo la carga que soportan por la tensión admisible de los cordones, para esfuerzos de compresión, normales a la dirección de las fibras. Si el ancho del rigidizador se hace igual a la anchura total de las cabezas, la dimensión mínima del rigidizador, en dirección paralela al vano, será:

$$
\mathrm{e}_{x}=\frac{\mathrm{P}}{\mathrm{W} \cdot \sigma_{c_{a d m}}}
$$

en la que: $P \quad=$ carga concentrada o reacciones.

$\mathrm{W}=$ ancho total de la viga.

$\sigma_{c_{a d m}}=$ tensión admisible a compresión perpendicular a las fibras.

$\mathrm{e}_{x} \quad=$ al espesor buscado del rigidizador.

En este caso, como el cortante admisible dado por el fabricante es $c=1.600 \mathrm{kp} ; \mathrm{W}=$ $=10 \mathrm{~cm}$ y $\sigma_{c_{a d m}}=19 \mathrm{kp} / \mathrm{cm}^{2}$ :

$$
e_{x}=\frac{1.600}{10 \times 19}=8,4 \mathrm{~cm}
$$

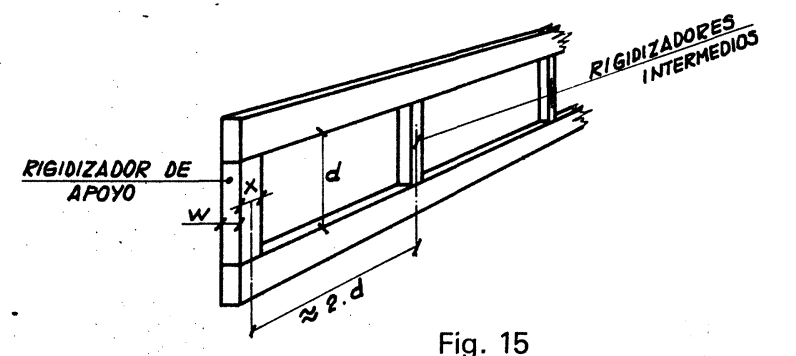

Fig. 15
- sea que las vigas DOKA H 36 deberian ir provistas de dos rigidizadores de cabeza o apoyo, junto al alma, de (10-3,1): $2=3,45 \mathrm{~cm}$ en dirección transversal al eje longitudinal de las vigas, y $8,4 \mathrm{~cm}$ en esa dirección indicada.

En cuanto a los rigidizadores intermedios, que sirven para evitar el pandeo del alma, sus dimensiones han de ser tales que puedan resistir los esfuerzos de compresión, que se pueden estimar equivalentes al esfuerzo cortante que soporta la viga, que corresponde a la sección en la que sitúa el rigidizador. Para el cálculo se supone que se comportan como pilares articulados en las cabezas o cordones de la viga. Es importante también que su espesor sea el necesario para realizar cómodamente la unión de los tableros. A continuación damos unas cuantas reglas prácticas, con las que facilitar el dimensionamiento de los rigidizadores: 
- El ancho del ridigizador debe ser igual al del cordón.

- Generalmente su espesor es inferior que el de los rigidizadores de cargas concentradas.

- Es conveniente que el espesor no sea inferior a $2,5 \mathrm{~cm}$.

- La separación entre rigidizadores es del orden del duplo de la distancia interna entre cordones (Fig. 15).

La separación entre rigidizadores intermedios depende: del espesor del alma; del espacio libre entre cordones; y de la tensión de cortadura sobre el alma de la viga.

Cuando la relación entre "d" (Fig. 15) y "t", el espesor del alma, es pequeña, como ocurre con la viga DOKA H 20, en que:

$$
\frac{d}{t}=\frac{122}{31}=3,9
$$

las vigas se pueden construir sin rigidizadores «intermedios»; pero cuando, como en la H 36 esa relación es elevada:

$$
\frac{\mathrm{d}}{\mathrm{t}}=\frac{254}{31}=8,2
$$

es indispensable colocar rigidizadores «intermedios", además de los de cabeza o extremos, con una separación $n$ da por la fórmula:

$$
n=\left(1+\frac{100-p}{25}\right)
$$

en la que:

- $n$ es la separación entre rigidizadores intermedios, cuando la tensión a cortadura del alma, es el $p \%$ de la admisible;

- $p$ es la distancia obtenida de la Fig. 8.

Aplicando la fórmula anterior al caso de la viga DOKA H 36, y suponiendo que el esfuerzo cortante en el alma no supera el máximo admisible, como debería ser, o sea que $p=100 \%$, como para un alma de $31 \mathrm{~mm}$ y una distancia libre entre cordones de $25,4 \mathrm{~cm}$ resulta por la Fig. 8 "p» $=13,5 \mathrm{~cm}$

$$
p=13,5 \times 1+\frac{100-100}{25}=13,5 \mathrm{~cm}
$$

luego nada menos que cada $13,5 \mathrm{~cm}$ tendrian que tener las vigas DOKA H 36 , los rigidizadores intermedios, y eso no rebasándose, como se rebasa ampliamente, en el alma la $\tau_{a d m}$ a cortadura.

\subsection{Cálculo de la unión del alma con los cordones}

En la Fig. 3.9 se plasma la sección transversal de la unión del alma con un cordón de la viga DOKA.

Como ya sabemos que para realizar una unión entallada, es preciso ejercer una notable presión entre las dos piezas a unir, tanto mayor cuanto menor es la longitud de la entalladura (véase gráfico 1 de la Parte VI) a DOKA se le ha tenido que presentar un grave problema a la hora de elegir el tipo de entalladura a emplear, pues la presión en los cordo- 


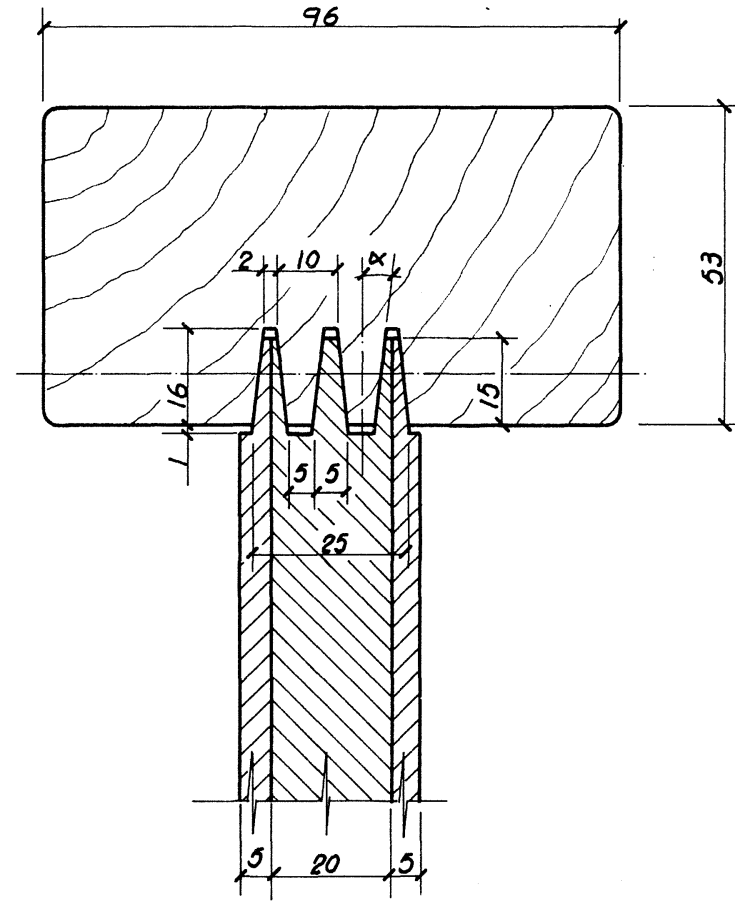

Fig. 16 nes es perpendicular a las fibras de la madera de éstos, y si elegía una entalla larga para disminuir la presión de armado, debilitaba notablemente la sección de aquéllos, mientras que eligiendo longitud corta, como lo ha hecho, la presión de armado es elevada y existe el peligro de que las cuñas del alma penetren en las entallas de los cordones y fisuren la madera.

En resumen: que se trata de una unión por entalladura difícil de realizar y que, como vamos a ver, no cumple las condiciones mínimas en este tipo de unión.

\subsubsection{Cálculo de la entalladura}

En la Fig. 3.9. se indica que la longitud de la entalladura es $L=15 \mathrm{~mm}$, y según la Fig. 1 de la Parte $\mathrm{VI}$ es necesario ejercer una presión de armado de nada menos que $97 \mathrm{kp} / \mathrm{cm}^{2}$,

cuando $\sigma_{c_{a d m}}=19 \mathrm{kp} / \mathrm{cm}^{2}$, luego es evidente el peligro de fisurar los cordones al armar la viga con esa presión de aprieto.

Las características de la unión entallada de la Fig. 16 son:

Longitud

Paso

Grueso extremo de la entalla

Pendiente

Ancho total de la unión

Juego del fondo de entalla

Grado de debilitamiento: $\beta / p$

Juego relativo de entalla: $S / L$
$\mathrm{L}=15 \mathrm{~mm}$

$\mathrm{p}=10 \mathrm{~mm}$

$\beta=2 \mathrm{~mm}$

$\alpha=10^{\circ}$

$\mathrm{H}=25 \mathrm{~mm}$

$\mathrm{S}=1 \mathrm{~mm}$

$v=0,2$

$\mathrm{j}=0,06 \mathrm{~mm}$

Aplicando la condición de Blömer para longitudes mayores de $10 \mathrm{~mm}$

$$
\mathrm{L}=4 \cdot \mathrm{p}(1-2 \cdot \mathrm{v})=4 \times 10(1-2 \times 0,2)=24 \mathrm{~mm}
$$

mientras que $L=15 \mathrm{~mm}$, luego la unión por entalladura DOKA no cumple esa condición. (Evidentemente por las razones expuestas anteriormente sobre el difícil compromiso entre la presión de armado, la longitud de entalla y el peligro de sobrepasar $\sigma_{c_{a d m}}$.

Siguiendo a Blömer, que fue quien calculó científicamente este tipo de uniones, la superficie necesaria en el plano de cola es:

$$
S_{c}=2 \cdot L \cdot(1-j)=1 \mathrm{~cm}^{2}
$$

condición que cumple ampliamente.

En cuanto a la pendiente, $\alpha=10^{\circ}$, o sea, $\operatorname{tg} \alpha=0,17632=1 / 5,7$ que no está dentro de los limites aconsejables explicados en la Parte VI, y que recordemos que oscilan entre $1 / 8$ y $1 / 10$, y la razón vuelve a ser la ya repetida de vitar una fuerte penetración de las cuñas del alma en las entallas de los cordones, para evitar que estos se fisuren, pues es evidente que cuanto mayor es el ángulo $2 \alpha$ de la cuña DOKA, mayor será la resistencia a la penetración en la entalla de los cordones, y así con una fuerte presión de armado, 
necesaria porque $L$ es pequena, se procura conjurar el peligro de fisuración de los cordones, al sobrepasar ampliamente $\sigma_{c_{a d m}}$.

En cuanto a la autorretención de la unión, y siguiendo aceptando, como en los dos casos de vigas anteriores, que el ángulo de rozamiento valga $18^{\circ}$ :

$$
2 \cdot \alpha=2 \times 10^{\circ}<2 \cdot \varrho_{o}=2 \times 18^{\circ}=36^{\circ}
$$

Pero nótese que no es un grado de autorretención muy elevado.

\subsubsection{Cálculo de tensiones rasantes en el alma}

Como es sabido por "Resistencia de Materiales», en cada sección de una viga, las tensiones rasantes son iguales y perpendiculares a las tensiones cortantes.

En el epígrafe 3.3. hemos calculado las tensiones cotantes, en base al cortante admisible $\mathrm{Q}_{\text {adm }}=1.600 \mathrm{kp}$ dado por el fabricante, $\mathrm{y}$, supuesto reparto uniforme, asciende a $\tau_{\max }=$ $=5 \mathrm{kp} / \mathrm{cm}^{2}$, luego la tensión rasante en la cara perpendicular será $\tau_{\max }=5 \mathrm{kp} / \mathrm{cm}^{2}$.

Pero por las experiencias hechas por el Laboratorio de Productos Forestales Americanos, se nota claramente cómo se puede apreciar comparando las Figs. 12, 13 y 14 que aun, cuando teóricamente las tensiones cortantes y las rasantes son máximas en la línea neutra, en la realidad, junto a los apoyos el reparto es muy diferente, y las tensiones máximas tienden a acercarse a la unión del alma con los cordones, es decir, en el caso que nos ocupa a la unión por entalladura, que ya hemos visto que no es correcta, lo que introduce otro factor deficiente en el proyecto y realización de esta viga que estudiamos.

Así se explica como en Alemania se ha comprobado que los extremos de la viga DOKA son sus puntos débiles, por donde comienzan a notarse despegues del alma con los cordones, porque según el reparto de $\tau_{\max }$ obtenido por los americanos (véase la Fig. 14), en los extremos esa $\tau_{\max }$ calculada antes, se sitúa peligrosamente cerca de la unión alma cordón, y producidos los primeros despegues por esa causa, por sus rendijas penetra la humedad y avanza en su acción destructora de la viga, y de ahi la corta vida útil que se ha comprobado en Alemania que tienen estas vigas, aparentemente sencillas de fabricar y económicas aparentemente, aunque en la realidad no sea asi por su excesivo peso, ya que el componente más importante del costo de una viga de madera para encofrado es su materia prima, o sea la madera.

\subsubsection{Pandeo del alma}

En realidad este tema ya se ha tratado ampliamente al hablar de los rigidizadores de cabeza o extremos y de los intermedios, en el epigrafe 3.3. Alma, donde se calcularon sus dimensiones y distancias, por lo que remitimos al lector a lo dicho en tal epígrafe.

\subsection{Resistencia a torsión}

Recordando cuanto dijimos en el epigrafe 2.6., relativo a la resistencia a torsión de la viga PERI, es aquí aplicable todo cuanto allí expusimos, de forma que con un error pequeño podemos admitir, que el momento torsor admisible es $1 / 3$ del flector dado por el fabricante, luego:

$$
\mathrm{M}_{t_{a d m}}=\frac{\sigma_{a d m}}{\sigma_{f_{a d m}}} \cdot \frac{\mathrm{M}_{f}}{3}=\frac{8}{124} \times \frac{1.700}{3}=36,5 \mathrm{~m} \cdot \mathrm{kp}
$$

valor algo superior al admisible para la viga PERI $(34 \mathrm{~m} \cdot \mathrm{kp})$, pero inferior al que permite para la viga STEIDLE-TREGAR $(41,3 \mathrm{~m} \cdot \mathrm{kp})$. 


\section{Vigas tipo Vierendeel}

Las vigas ACROW-36 se fabrican en dos longitudes; 2.700 y $3.720 \mathrm{~mm}$ y son de tipo Vierendeel, como se muestra en las figuras.

La viga de $2.700 \mathrm{~mm}$ pesa $21,6 \mathrm{kp}$ y la de $3.720 \mathrm{~mm} 80 \mathrm{kp}$, por lo que los pesos unitarios respectivos son: 8 y $8,06 \mathrm{kp} / \mathrm{m}$, de los que tomaremos $8 \mathrm{kp} / \mathrm{m}$, peso bastante elevado para este tipo de vigas de madera, como ya se ha visto en el Cuadro $n .^{\circ} 3$ de la Parte 1 de este trabajo publicada en el n. ${ }^{\circ} 318$ de esta Revista.

\subsection{Características mecánicas}

Tales vigas, según su fabricante, tienen las siguientes características:

$$
\begin{aligned}
\mathrm{M}_{u l m} & =1.350 \mathrm{~m} \cdot \mathrm{kp} \\
\mathrm{Q}_{u l m} & =1.700 \mathrm{kp}
\end{aligned}
$$

luego sus resistencias específicas respecto a su peso son:

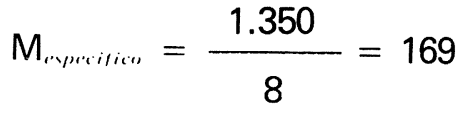

$$
\begin{aligned}
& \mathrm{Q}_{\text {(“,mecificio }}=\frac{1.700}{8}=212
\end{aligned}
$$

Los cordones de estas vigas están formados por dos largueros de $35 \times 95 \mathrm{~mm}$, encolados a tableros de $13 \times 170 \times 360 \mathrm{~mm}$, dando lugar a una viga de sección en I, dibujada y acotada en la Fig. 19, cuya sección da un momento de inercia máximo de:

$$
I_{x}=24.348 \mathrm{~cm}^{4}
$$

Este tipo de viga tiene dos inconvenientes, uno de concepto o proyecto y otro de construcción. El primero hace difícil y pesado el cálculo minucioso de la viga por ser hiperestática, y sobre todo cuando es preciso averiguar con cierto grado de exactitud la flecha que puede originar bajo carga.

El segundo porque es peligroso confiar la unión de los cordones a los montantes a la cola, y parece que ningún experimentado constructor de armaduras de madera, para trabajar a intemperie y resistir cargas a veces fuertes, aprobará fácilmente tal construcción.

Efectivamente: en la Obra "Cálculo de Estructuras de Madera", del Catedrático de la Escuela de Ingenieros de Montes de Madrid, don Ramón Argüelles Alvarez, en la pág. 136 se hace referencia a este tipo de construcción, especificando que los cordones deben unirse al alma (montantes separados en este caso) por medio de clavos con surcos de espiral, con lo que se pierde el carácter más notable de las vigas de madera encolada para encofrados modernos, que es precisamente la ausencia total de medios de unión metálicos; de plástico; etc., es decir, medios que no sean mediante labrados especiales en la madera, más o menos ingeniosos asegurados por medio de colas modernas sintéticas, como hemos visto en las Partes $\mathrm{V}$ y VI.

También en dicha obra se hace referencia a los rigidizadores del alma para evitar su pandeo local o alabeo (Págs. 136 y 175), y se mencionan los rigidizadores de apoyo (pág. 191), de todo lo cual carece este tipo de viga, tal como figura en los catálogos del fabricante.

\subsection{Cálculo de la viga mediante ordenador}

Como ya hemos dicho que el cálculo manual de una viga Vierendeel es difícil, en este caso hemos recurrido al Centro de Proceso de Datos del Ministerio de Obras Públicas, agradeciendo aquí al Dr. Ing. de Caminos don Avelino F. Samartín, su valiosa colaboración. 
En la Fig. 20 aparece el esquema de cargas y apoyos que luego se realizó en el ensayo hecho en el Instituto Torroja, que también es justo agradecer la colaboración de los Dres. Ings. de Caminos don Rafael Fernández y don José Quereda que dirigieron y realizaron el ensayo respectivamente.

El caso de viga calculada y comprobada fue el de $2.700 \mathrm{~mm}$ de longitud, y en la Fig. 21 figura el esquema de cargas reales, habida cuenta del ancho de montantes, numerándose los nudos con números correlativos y las barras también, pero estos encerrándolos en círculos para distinguirlos de los anteriores.

La separación óptima entre apoyos debe ser:

$$
\begin{aligned}
& \ell_{1}=0,207 \cdot \mathrm{L}=559 \mathrm{~mm} \\
& \ell_{2}=0,586 \cdot \mathrm{L}=1.582 \mathrm{~mm}
\end{aligned}
$$

Se calcula la viga con apoyo en el nudo 10 (A) y se supone una carga de valor total uniformemente repartida de $p=1 \mathrm{kp} / \mathrm{mm}$, que se concentra en los montantes en cargas puntuales de valor $P=170 \mathrm{kp}$.

Las barras tienen las siguientes características mecánicas:

$$
\begin{aligned}
& \text { - Cordones: } A=96 \times 58=5.568 \mathrm{~mm}^{2} ; I=\frac{96^{3} \times 58}{12}=4.276 .224 \mathrm{~mm}^{4} \\
& \text { - Montantes: } \mathrm{A}=170 \times 18=3.060 \mathrm{~mm}^{2} ; \mathrm{I}=\frac{170^{3} \times 18}{12}=7.369 .500 \mathrm{~mm}^{4}
\end{aligned}
$$

La unión de la junta de los nudos se ha simulado mediante una inercia suficientemente alta, concretamente $I=999.999 \times 10^{3}$ y el módulo de elasticidad adoptado (ficticio) $E=10^{6} \mathrm{Mp} / \mathrm{m}^{2}=10^{3} \mathrm{kp} / \mathrm{mm}^{2}$.

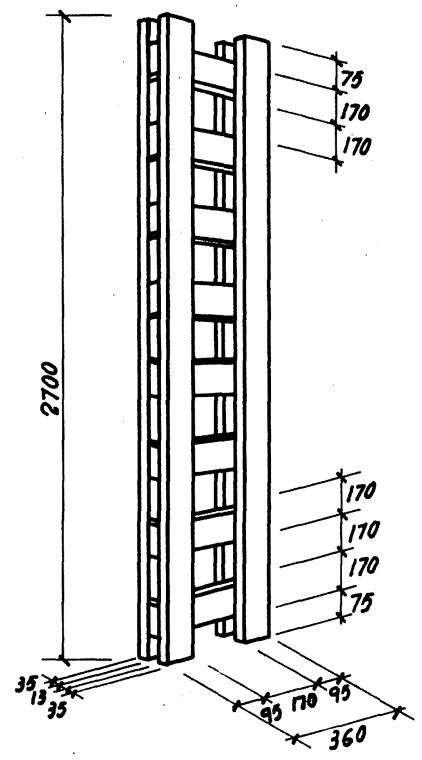

Fig. 17

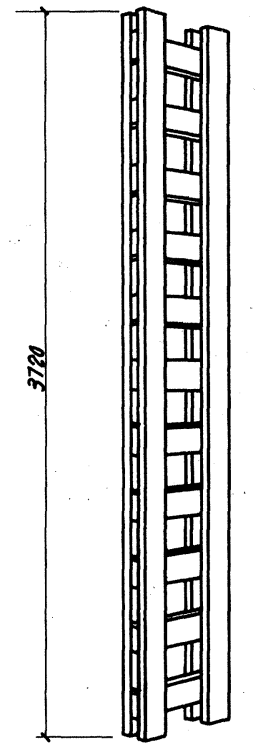

Fig. 18

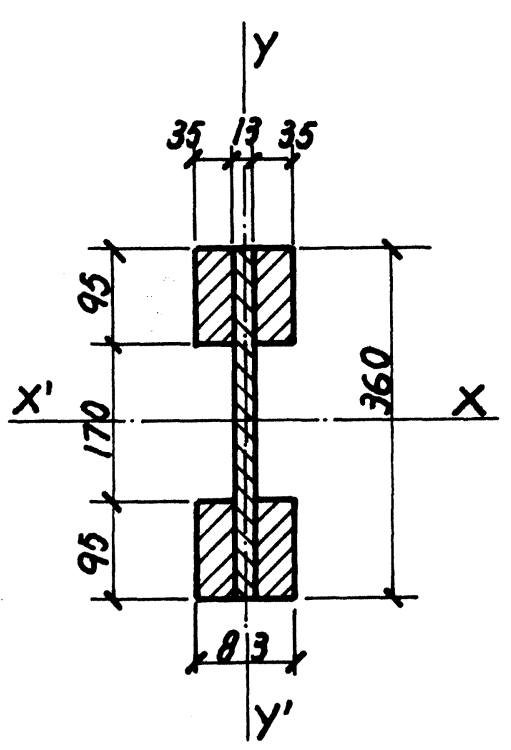

Fig. 19 


\section{Loading number 1}

\section{MEMBER FORCES}

\begin{tabular}{|c|c|c|c|c|}
\hline MEMBER & JOINT & AXIAL FORCE & SHEAR FORCE & MOMENT \\
\hline 1 & 1 & -0.004 & -0.056 & -2.10 \\
\hline 1 & 3 & 0.004 & 75.056 & -2814.62 \\
\hline 2 & 3 & -0.005 & -72.924 & 2735.18 \\
\hline 2 & 5 & 0.005 & 72.924 & -8933.72 \\
\hline 3 & 5 & -92.910 & 68.074 & 10393.02 \\
\hline 3 & 7 & 92.910 & -68.074 & -4606.72 \\
\hline 4 & 7 & -93.489 & 69.981 & 4464.93 \\
\hline 4 & 9 & 93.489 & 100.018 & -7018.09 \\
\hline 5 & 9 & -85.521 & -100.437 & 7359.23 \\
\hline 5 & 11 & 85.521 & 100.437 & -15896.40 \\
\hline 6 & 11 & 504.121 & 518.107 & 83812.70 \\
\hline 6 & 13 & -504.121 & -518.107 & -39773.57 \\
\hline 7 & 13 & 503.557 & 517.259 & 39745.35 \\
\hline 7 & 15 & -503.557 & -347.259 & 33738.72 \\
\hline 8 & 15 & 632.300 & 345.069 & -33946.78 \\
\hline 8 & 17 & -632.300 & -345.069 & 63277.67 \\
\hline 9 & 17 & 1217.391 & 270.219 & 20.657 .85 \\
\hline 9 & 19 & -1217.391 & -270.219 & 2310.83 \\
\hline 10 & 19 & 1198.548 & 265.589 & -2826.68 \\
\hline 10 & 21 & -1198.548 & -95.589 & 33526.83 \\
\hline 11 & 21 & 1187.442 & 93.356 & -34643.83 \\
\hline 11 & 23 & -1187.442 & -93.356 & 42579.11 \\
\hline 12 & 23 & 1366.193 & 82.738 & -13495.28 \\
\hline 12 & 25 & -1366.193 & -82.738 & 20528.07 \\
\hline 13 & 25 & 1357.477 & 82.945 & -21135.08 \\
\hline 13 & 27 & -1357.477 & 2.054 & 24572.97 \\
\hline 14 & 2 & 0.002 & -0.033 & -1.10 \\
\hline 14 & 4 & -0.002 & 0.033 & -1.39 \\
\hline 15 & 4 & 0.003 & 4.645 & 148.10 \\
\hline 15 & 6 & -0.003 & -4.645 & 246.71 \\
\hline 16 & 6 & 104.301 & -315.679 & -26582.24 \\
\hline 16 & 8 & -104.301 & 315.679 & -250.51 \\
\hline 17 & 8 & 103.943 & -312.593 & 102.43 \\
\hline 17 & 10 & -103.943 & 312.593 & -53243.39 \\
\hline 18 & 10 & 106.173 & 1140.658 & 53246.86 \\
\hline 18 & 12 & -106.173 & -1140.658 & 43709.07 \\
\hline 19 & 12 & -496.841 & 348.482 & 47954.58 \\
\hline 19 & 14 & 496.841 & -348.482 & -18333.57 \\
\hline 20 & 14 & -492.515 & 344.864 & 18424.90 \\
\hline 20 & 16 & 492.515 & -344.864 & 40201.98 \\
\hline 21 & 16 & -193.784 & 335.283 & -40262.89 \\
\hline 21 & 18 & 193.784 & -335.283 & 68761.95 \\
\hline 22 & 18 & -918.749 & 205.924 & 13556.29 \\
\hline 22 & 20 & 918.749 & -205.924 & 3947.25 \\
\hline$\overline{23}$ & 20 & -923.132 & 194.722 & -3342.32 \\
\hline 23 & 22 & 923.132 & -194.722 & 36445.17 \\
\hline 24 & 22 & -925.346 & 177.224 & -37349.43 \\
\hline 24 & 24 & 925.346 & -177.224 & 52413.49 \\
\hline 25 & 24 & -1137.258 & 2.186 & -23565.37 \\
\hline 25 & 26 & 1137.258 & -2.186 & 23751.21 \\
\hline 26 & 26 & -1133.715 & 0.000 & -23112.55 \\
\hline 26 & 28 & 1133.715 & -0.000 & 23112.60 \\
\hline 27 & 6 & 315.965 & 95.385 & 26518.52 \\
\hline 27 & 5 & -315.965 & -95.385 & -1336.71 \\
\hline 28 & 12 & 789.894 & -604.522 & -91706.48 \\
\hline 28 & 11 & -789.894 & 604.522 & -67887.45 \\
\hline 29 & 18 & 113.895 & -624.669 & -81539.43 \\
\hline 29 & 17 & -113.895 & 624.669 & -83373.21 \\
\hline 30 & 24 & 175.022 & -223.024 & -29264.36 \\
\hline 30 & 23 & -175.022 & 223.024 & -29614.08 \\
\hline
\end{tabular}


APPLIED JOINT LOADS, FREE JOINTS

\begin{tabular}{|c|c|c|c|}
\hline JOINT & FORCE $X$ & FORCE Y & MOMENT Z \\
\hline 1 & -0.004 & -0.056 & -2.10 \\
\hline 2 & 0.002 & -0.033 & -1.10 \\
\hline 3 & -0.001 & 2.132 & -79.43 \\
\hline 4 & 0.001 & 4.678 & 146.70 \\
\hline 5 & 2.481 & -174.966 & 122.58 \\
\hline 6 & 8.912 & -4.359 & 183.00 \\
\hline 7 & -0.578 & 1.907 & -141.79 \\
\hline 8 & -0.357 & 3.085 & -148.07 \\
\hline 9 & 7.968 & -0.418 & 341.13 \\
\hline 11 & -14.879 & -171.349 & 28.84 \\
\hline 12 & 1.506 & -2.281 & -42.82 \\
\hline 13 & -0.564 & -0.848 & -28.22 \\
\hline 14 & 4.326 & -3.618 & 91.32 \\
\hline 15 & 128.742 & -2.190 & -208.06 \\
\hline 16 & 298.730 & -9.580 & -60.90 \\
\hline 17 & -39.577 & -188.745 & 562.32 \\
\hline 18 & -100.295 & -15.463 & 778.79 \\
\hline 19 & -18.843 & -4.630 & -515.84 \\
\hline 20 & -4.383 & -11.201 & 604.93 \\
\hline 21 & -11.105 & -2.232 & -1117.00 \\
\hline 22 & -2.213 & -17.498 & -904.25 \\
\hline$\overline{23}$ & -44.273 & -185.640 & -530.26 \\
\hline 24 & 11.112 & -0.015 & -416.25 \\
\hline 25 & -8.716 & 0.207 & -607.01 \\
\hline 26 & 3.543 & -2.185 & 638.66 \\
\hline
\end{tabular}

FREE JOINT DISPLACEMENTS

\begin{tabular}{|c|c|c|c|}
\hline JOINT & X-DISPLAC. & Y-DISPLAC. & ROTATION \\
\hline 1 & 0.1420 & 0.3733 & -0.0011 \\
\hline 2 & -0.1101 & 0.3217 & -0.0006 \\
\hline 3 & 0.1420 & 0.2895 & -0.0011 \\
\hline 4 & -0.1101 & 0.2744 & -0.0006 \\
\hline 5 & 0.1420 & 0.1935 & -0.0011 \\
\hline 6 & -0.1101 & 0.2207 & -0.0006 \\
\hline 7 & 0.1434 & 0.0974 & -0.0011 \\
\hline 8 & -0.1117 & 0.1672 & -0.0006 \\
\hline 9 & 0.1463 & -0.1045 & -0.0012 \\
\hline 11 & 0.1476 & -0.2118 & -0.0012 \\
\hline 12 & -0.1165 & -0.1437 & -0.0016 \\
\hline 13 & 0.1399 & -0.3195 & -0.0012 \\
\hline 14 & -0.1090 & -0.2875 & -0.0016 \\
\hline 15 & 0.1245 & -0.5787 & -0.0012 \\
\hline 16 & -0.0939 & -0.5716 & -0.0012 \\
\hline 17 & 0.1148 & -0.6884 & -0.0012 \\
\hline 18 & -0.0910 & -0.6785 & $-0,0012$ \\
\hline 19 & 0.0962 & -0.7980 & -0.0012 \\
\hline 20 & -0.0769 & -0.7853 & -0.0012 \\
\hline 21 & 0.0597 & -0.9649 & -0.0004 \\
\hline 22 & -0.0487 & -0.9503 & -0.0004 \\
\hline 23 & 0.0415 & -1.0048 & -0.0004 \\
\hline 24 & -0.0346 & -0.9897 & -0.0004 \\
\hline 25 & 0.0207 & -1.0445 & -0.0004 \\
\hline 26 & -0.0173 & -1.0288 & -0.0004 \\
\hline
\end{tabular}

SUPPORT JOINT DISPLACEMENTS

REACTIONS. APPLIED LOADS SUPPORT JOINTS

\begin{tabular}{|c|c|c|c|}
\hline JOINT & X-DISPLAC. & Y.DISPLAC. & ROTATION \\
\hline 10 & -0.1149 & 0.0000 & -0.0016 \\
\hline 27 & 0.0000 & -1.0648 & 0.0000 \\
\hline 28 & 0.0000 & -1.0483 & 0.0000 \\
\hline
\end{tabular}

Los resultados, que se recogen en las páginas siguientes, hechos por el ordenador del referido Centro de Proceso de Datos del Ministerio de Obras Públicas son para las barras horizontales más desfavorables:

$$
\begin{array}{ll}
\text { - Barra (18); Nudo } 10 & \\
M=532,5 \mathrm{~cm} \cdot \mathrm{kp} & \sigma_{f}=59,7 \mathrm{kp} / \mathrm{cm}^{2} \\
\mathrm{~N}=106,2 \mathrm{kp} & \sigma_{c}=2,0 \mathrm{kp} / \mathrm{cm}^{2} \\
\mathrm{Q}=1.140,6 \mathrm{kp} & \tau=30,7 \mathrm{kp} / \mathrm{cm}^{2}
\end{array}
$$

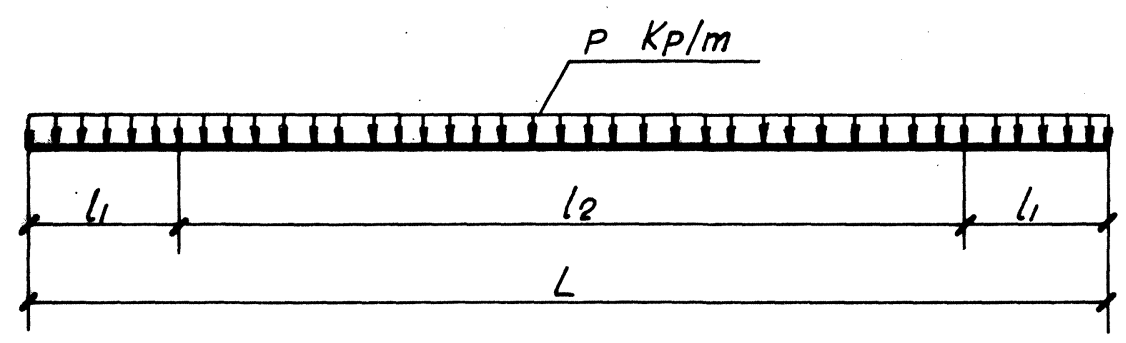

Fig. 20

\subsection{Conclusiones del cálculo}

- Barra (13); nudo 25

$$
\begin{array}{llr}
M=221,35 \mathrm{~cm} \cdot \mathrm{kp} & \sigma_{f}=23,7 \mathrm{kp} / \mathrm{cm}^{2} \\
\mathrm{~N}=1.357 \mathrm{kp} & \sigma_{c}=24,3 \mathrm{kp} / \mathrm{cm}^{2} \\
\mathrm{Q}=83 \mathrm{kp} & \tau=2,2 \mathrm{kp} / \mathrm{cm}^{2}
\end{array}
$$


y para las barras verticales la más cargada es:

- Barra (28); nudo 12

$$
\begin{array}{ll}
\mathrm{M}=91,706 \mathrm{~cm} \cdot \mathrm{kp} & \sigma_{f}=105,7 \mathrm{kp} / \mathrm{cm}^{2} \\
\mathrm{~N}=790 \mathrm{kp} & \sigma_{c}=25,8 \mathrm{kp} / \mathrm{cm}^{2} \\
\mathrm{Q}=605 \mathrm{kp} & \tau=29,6 \mathrm{kp} / \mathrm{cm}^{2}
\end{array}
$$

Se deduce del cálculo que la barra más cargada a flexión es la vertical o montante $28 \quad \mathrm{Y}$ recordando las tensiones admisibles especificadas en el epigrafe 1.1. y según Norma

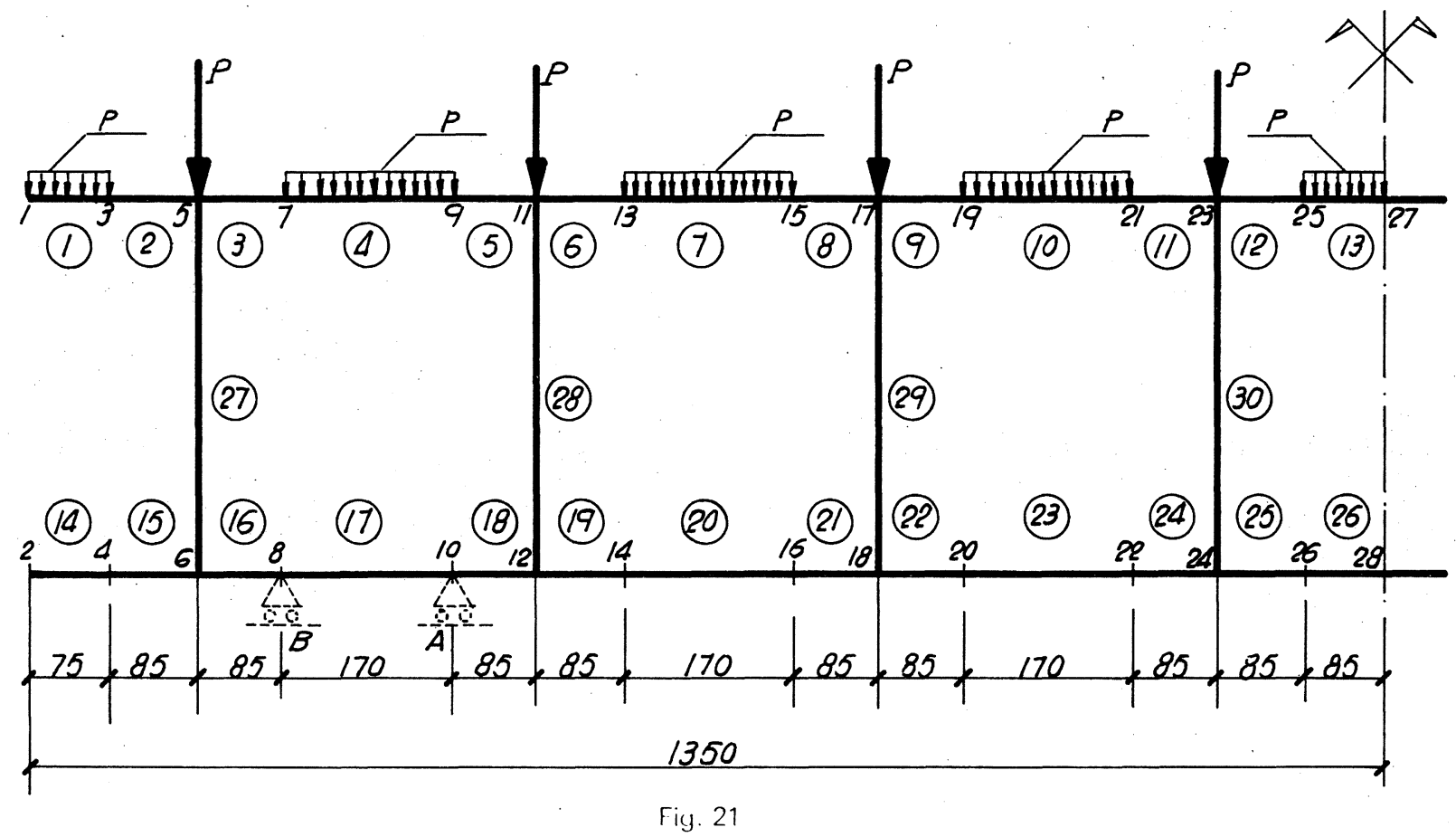

DIN 1052, disminuidas a sus $5 / 6$ por estar la madera de las vigas en contacto con la humedad, pero aumentadas en un $15 \%$ porque se emplea tipo de carga $\mathrm{HZ}$, es decir, las principales y las accesorias resultan ser:

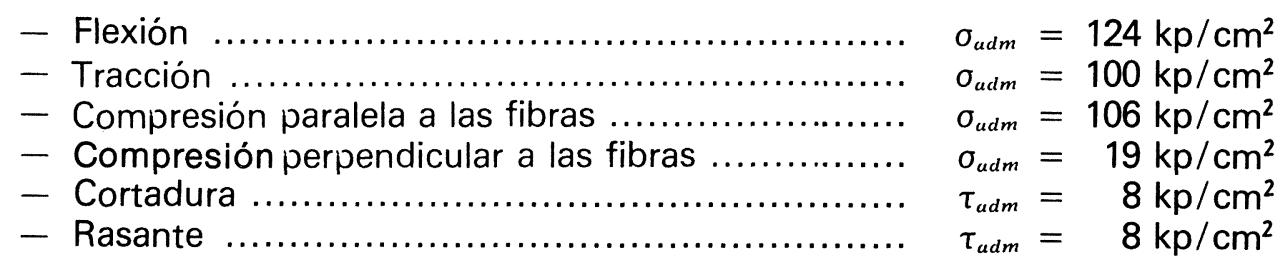

De las tensiones admisibles anteriores se deduce que la barra (28) en su nudo 12, o sea prácticamente en el apoyo (véase las Figs. 21 y 22) resiste perfectamente a la flexión y a la tracción a que está sometida, pero en cambio no así al esfuerzo rasante de $\tau=29,6 \mathrm{kp} / \mathrm{cm}^{2}$.

En cuanto a la barra horizontal o cordón inferior (18) en su nudo 10 tampoco puede soportar el esfuerzo cortante de $30,7 \mathrm{kp} / \mathrm{cm}^{2}$ determinado por el cálculo.

Esa falta de resistencia de la barra (18) al esfuerzo rasante en su nudo 10 , o sea en el apoyo A, Figs. 21 y 22 se ve perfectamente corroborada por el ensayo a rotura, hecho en el Instituto Eduardo Torroja.

Modernamente, en América, para determinar las tensiones admisibles, se parte de unas tensiones básicas, deducidas a partir de probetas sin defectos ensayadas en condiciones óptimas, y a partir de valores obtenidos, se calculan las tensiones admisibles introducción reducciones por defectos y otras causas de la forma siguiente: 
1) La tensión última media se reduce en un $25 \%$ para contar con la posible dispersión de los resultados.

2) El valor obtenido anteriormente se disminuye en un 43,8 \%, para tener en cuenta la disminución de la tensión de agotamiento, como consecuencia de la posible aplicación de cargas prolongadas.

3) El valor deducido en 2) se reduce en un $40 \%$, para conseguir un coeficiente de seguridad no inferior a $5 / 3$, el cual puede variar según el tipo de construcción y el trato que reciba.

4) Finalmente, ese resultado se minora o mayora en un porcentaje que varía en función del comportamiento de la madera de que se trate cuando se utiliza como viga. Normalmente ese porcentaje suele disminuir la resistencia en un $10 \%$ según la experiencia que se posea.

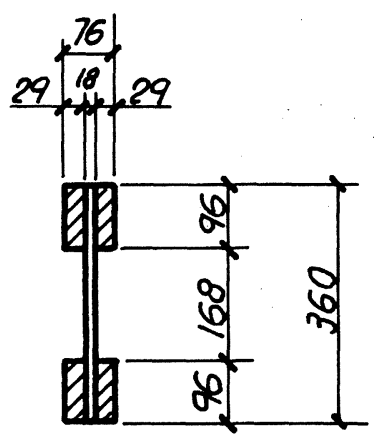

SECCION

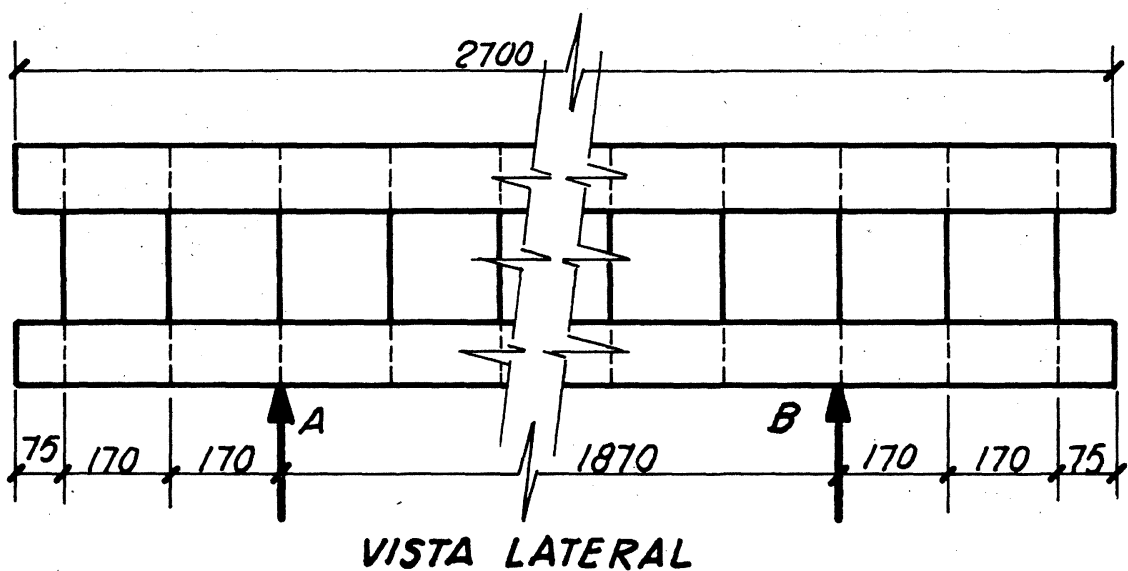

Fig. 22

Sin tener en cuenta la reducción 4), la tensión admisible es 1/4 de la básica, luego el coeficiente de seguridad en tal hipótesis es $v=4$. Pero contando con la reducción 4) tal coeficiente de seguridad aumenta a $v=4,5$.

Pues bien: si aplicamos esos dos límites obtenidos para el coeficiente de seguridad, con que se calculan las vigas de madera, a las tensiones admisibles antes especificadas y concretamente la tensión rasante, causante de la rotura de la viga tipo Vierendeel ensayada. similar a la fabricada por ACROW-WOLFF, llegaremos a la conclusión de que la barra (18) en su nudo 10, o sea en el apoyo tenia que romperse, con la carga supuesta en el cálculo anterior de $1 \mathrm{kp} / \mathrm{mm}=1 \mathrm{Mp} / \mathrm{m}$, porque:

$$
\tau_{u d m}=8 \mathrm{kp} / \mathrm{cm}^{2}=\tau_{a d m}
$$

o sea: limite mínimo $\tau_{a d m} \times 4=32 \mathrm{kp} / \mathrm{cm}^{2}>30,7 \mathrm{kp} / \mathrm{cm}^{2}$ límite máximo $\tau_{a d m} \times 4,5=36 \mathrm{kp} / \mathrm{cm}^{2}>30,7 \mathrm{kp} / \mathrm{cm}^{2}$

luego como se ve la carga supuesta produce la ruina por esfuerzo cortante en la citada barra (18) en su nudo 10, como comprobaremos en el ensayo a rotura más adelante.

\subsection{Resistencia a torsión}

En el epígrafe 2.6. relativo al momento torsor admisible para una viga de madera de sección en doble $T$, ya dijimos que, según Timoshenko, con pequeño error del orden de un $4 \%$ se puede admitir que el $M_{t_{a d m}}=1 / 3 \cdot M_{j_{a d m}}$, y como el fabricante de estas vigas tipo Vierendeel (ACROW-WOLFF) da $M_{j_{a d m}}=1.350 \mathrm{~m} \cdot \mathrm{kp}$, el torsor admisible sería 


$$
\mathrm{M}_{t_{\text {adm }}}=\frac{\tau_{a d m}}{\sigma_{f_{u d m}}} \cdot \frac{\mathrm{M}_{f_{u d m}}}{3}=\frac{8}{124} \times \frac{1.350}{3}=29 \mathrm{~m} \cdot \mathrm{kp} .
$$

Pero seguramente el valor real será menor por la discontinuidad del alma, que está formada, como se sabe, sólo por montantes espaciados entre ellos igual dimensión que su ancho.

\subsection{Ensayo a rotura}

Para confirmar, como en los casos de vigas anteriores, los resultados de los cálculos teóricos, en donde casi siempre hay que introducir hipótesis simplificadoras, que lógicamente deforman aquéllos, en cantidad dependiente del acierto de tales hipótesis, presentamos seguidamente los resultados de un ensayo hecho por el Instituto Eduardo Torroja, sobre una viga de las dimensiones especificadas en el mismo y el de la Fig. 22 ligeramente diferentes en cuanto a cordones y montantes, con las dadas por el fabricante en su catálogo e indicadas en las Figs. 17 y 19, lo cual es accesorio y no afecta al fondo del tema.

\subsubsection{Resultado del ensayo a rotura}

En el Informe que sigue se dice que «la viga se rompió por el montante del extremo más próximo al flexímetro F-1 para una carga total de $3.350 \mathrm{kp}$ ".

Como el Autor estuvo presente en el ensayo está obligado a matizar un poco la frase anterior, pues lo cierto es que cuando se llegó a una carga global de $3.000 \mathrm{kp}$, ya se presentaron claros síntomas de ruina inminente, pero se siguió cargando para romper totalmente la viga, la cual desde esos $3.000 \mathrm{kp}$ ya no sea viga como tal, dado el alabeo que tenía y las fisuras que se apreciaban en la sección de rotura del montante de apoyo.

Prueba de ser eso así, es que el Ingeniero examinador y director del ensayo, perteneciente a la plantilla del referido Instituto, señor don José Quereda, que tomaba los datos de las fuerza del gato de carga, de las lecturas de los flexímetros y de la escalilla, para poder confeccionar el Cuadro n. ${ }^{\circ} 1$, dio éste por terminado para un cortante en cada apoyo y a cada lado del mismo de $750 \mathrm{kp}$, o sea cuando el gato de carga estaba en $3.000 \mathrm{kp}$, así que en realidad la viga ensayada comenzó su ruina para una carga uniforme de:

$$
\frac{3.000}{2,7}=1.111 \mathrm{kp} / \mathrm{m}
$$

que sólo difiere en $111 \mathrm{kp} / \mathrm{m}$, o sea un $11,1 \%$ de la carga de cálculo $(1.000 \mathrm{kp} / \mathrm{m})$ supuesta en el epígrafe 4.2.

Como por la fotografía $n .{ }^{\circ} 3$ del Informe del mencionado Instituto, se ve que la rotura de la viga sobrevino por exceso de tensión rasante en el montante de apoyo, cosa que ya se dedujo previamente por cálculo, vamos a comprobar el valor de esa $\tau$, para ver su concordancia con la obtenida en los cálculos anteriores.

Se sabe que el esfuerzo rasante vale:

$$
\tau=\frac{c \cdot m}{1 \cdot b}(1)
$$

en la que $C$ es el cortante vertical, en nuestro caso $750 \mathrm{kp}$; $\mathbb{M}$ el momento estático de la superficie rota respecto a su línea neutra:

$$
\tau=\frac{1,8 \times 17^{2}}{8}=65 \mathrm{~cm}^{3}
$$


I, el momento de inercia de esa sección respecto del mismo eje:

$$
I=\frac{1}{12} \times 1,8 \times 17^{3}=737 \mathrm{~cm}^{4}
$$

y $\mathrm{b}$ el ancho de la sección arruinada, o sea, $1,8 \mathrm{~cm}$. Sustituyendo en la expresión (1) resulta:

$$
\tau=\frac{750 \times 65}{737 \times 1,8}=36,7 \mathrm{kp} / \mathrm{cm}^{2}
$$

cifra casi igual a la calculada en el epígrafe 4.3., cuando adoptamos un coeficiente de seguridad $v=4,5$.

Queda pues comprobada la casi total concordancia de los cálculos con la realidad de los ensayos.

\subsubsection{Coeficiente de seguridad}

De la comparación hecha en el apartado anterior, entre los resultados de los cálculos y de los ensayos, y habida cuenta que una viga Vierendeel es más deformable que las de celosía - alma llena, adoptamos definitivamente como coeficiente de seguridad $v$ para este tipo de viga, el mayor de los obtenidos por el método americano (epígrafe 4.3.) o sea 4,5.

\subsubsection{Tensiones admisibles de trabajo}

Siguiendo el citado método americano para determinar las tensiones admisibles, se construyó una Viga Vierendeel, análoga a las catalogadas por ACROW-WOLFF, empleando en los cordones superior e inferior, Pino de Suecia, exento casi de nudos y los pocos que tenía eran pequeños y no saltadizos, calidad US con un $20 / 25 \%$ de $5^{\text {a }}$ y $12 \%$ de humedad.

Los montantes se construyeron a base de tablero aglomerado, que fue encolado a los largueros de que consta cada cordón, mediante cola sintética nacional de fenol-formaldehido, conocida en el comercio con el nombre de Cascofen PA-8, y prensados en un banco de armar accionado por bombines neumáticos, y para mayor seguridad en cada nudo de montante con largueros de cordón, se clavaron mecánicamente dos clavos que abarcaban toda la profundidad del nudo o unión.

De la fig. 3. deducimos que el momento de inercia es:

$$
I=\frac{1}{12} \cdot 1,3 \times 36^{3}+4 \times 3,5 \times 9,5 \times 13,25^{2}=28.400 \mathrm{~cm}^{4}
$$

y el módulo resistente:

$$
W^{\prime}=\frac{\mathrm{l}}{\mathrm{h} / 2}=\frac{28.400}{18}=1.578 \mathrm{~cm}^{3}
$$

Pero en las secciones donde no existe montante esos valores son:

$$
I^{\prime \prime}=23.350 \mathrm{~cm}^{4} \text { y } W^{\prime \prime}=1.297 \mathrm{~cm}^{3}
$$

Tomando una media tendremos:

$$
\mathrm{I}=25.875 \mathrm{~cm}^{4} \text { y W }=1.437 \mathrm{~cm}^{3}
$$


Como los cordones trabajan a tracción o compresión, tomando la tensión admisible menor de las dos que es

$\sigma_{t_{a d m}}=100 \mathrm{kp} / \mathrm{cm}^{2}$ resulta un momento admisible de:

$$
\mathbf{M}_{\text {adm }}=1.437 \times 100=1.437 \mathrm{~m} \cdot \mathrm{kp}>1.350
$$

dado por el fabricante.

Pero donde no comprendemos como el fabricante puede dar $1.700 \mathrm{kp}$ es para el cortante. En efecto: El momento estático de la sección de la Fig. 3. vale:

$$
M_{c}=1,3 \times 18 \times 9+2 \times 3,5 \times 9,5 \times 13,25=1.092 \mathrm{~cm}^{3}
$$

y como

$$
\begin{gathered}
\tau=\frac{\mathrm{Q} \cdot \mathrm{m}}{\mathrm{l} \cdot \mathrm{b}} \quad \mathrm{Q}=\frac{8 \cdot \mathrm{l} \cdot 1,3}{\mathrm{M}_{e}} \\
\mathrm{Q}_{u l m}=\frac{8 \times 25.875 \times 1,3}{1.092}=246 \mathrm{kp}
\end{gathered}
$$

resultando lógico, que del ensayo hecho por el Instituto Eduardo Torroja, a $Q=750 \mathrm{kp}$ la viga comienza a romperse y ese valor de $\mathrm{Q}_{a d m}$ obtenido supone, respecto del ensayo hecho un coeficiente de seguridad de:

$$
v=\frac{750}{246}=3
$$

un poco bajo para nuestro parecer en este tipo de viga. Ahora bien, si nos atenemos a la carga total de ruina dada por los firmantes del ensayo, entonces el cortante de rotura será:

$$
\frac{3.350}{4}=837,5 \mathrm{kp}
$$

y el coeficiente de seguridad llegará a:

$$
v=\frac{837,5}{246}=3,4
$$

que aún nos parece bajo para esta clase de viga.

\section{résumé}

ETUDE SUR LES COFFRAGES EN BOIS MODERNES

Juan M. de la Peña Aznar,

Dr. ingénieur industriel

L'auteur continue à traiter le thème sur les "Coffrages en bois modernes", résumant l'étude comparative - contenue dans la Partie III- des différentes poutres en bois collé existant sur le marché et donnant, en plus, les charges et les contraintes admissiplus, les charges et les contraintes admissibles pour les bois coniferes et la proposition de reglementalic Bois de l'Institut Forestier de Recherches et d'Expériences d'Espagne.

Dans la partie $\mathbf{V}$ du statut, ici publiée, l'auteur aborde le sujet important des colles utilisées pour les assemblages en bois, ce qui est réellement vital pour l'obtention des poutres en bois en treillis simplement collées.

\section{summary}

\section{STUDY ON MODERN WOODEN FORMS}

Dr. Juan M. de la Pena Aznar, Industrial Engineer

The author continues the development of the subject "Modern Timber Formwork", by summing up the comparative examination -already carried out in Part III of this Study - of the different types of glued timber existing on the market. In addition, the loads and stresses allowable for coniferous loads and stresses allowable for coniferous timber and the proposal for establishing Re-
gulations for the Timber Section of the Research and Experimental Forestry Institute of Spain are given.

In part $V$ of the author's Study, published in this article, the important subject of the glues used for joining timber, a truly vital glues used for joining timber, a truly vital
point in order to obtain louvered timber point in order to obtain louvered timber
beams which are simply glued together, is approached.

\section{zusammenfassung}

\section{STUDIE UBER MODERNE HOLZ- VERSCHALUNGEN}

Dr. Juan M. de la Peña Aznar, Industrieingenieur

Der Verfasser entwickelt weiterhin das The ma der "Modernen Holzverschalungen" und fasst die Vergleichsstudie - welche bereits in Teil III dieser Arbeit aufgestellt wurde der verschiedenen, handelsüblichen Holzverschalungen zusammen. Er gibt dabei gleichzeitig zulässige Belastungen und Spannungen für Nadelhölzer und einen Vorschlag für eine Verordnung der Abteilung für Hölzer des Spanischen Forstwirtschaftsinstitutes für Forschung und Versuchsdurchführung an.

In Teil $V$ der Studie, welche in diesem Artikel veröffentlicht wird, werden die bedeutenden Leimsorten behandelt, die bei der $\mathrm{Holz}$ verbindung eingesetzt werden und bei de Herstellung von einfach geleimten Holzbalken lebenswichtig sind. 